\title{
Bovine serum albumin-imprinted magnetic poly(2-pyrrolidone) microparticles for protein recognition
}

\author{
Nadya V. Dencheva, Filipa D. Oliveira, Joana F. Braz, Zlatan Z. Denchev* \\ IPC - Institute for Polymers and Composites, Department of Polymer Engineering, University of Minho, Guimaraes 4800-056, Portugal
}

\section{A R T I C L E I N F O}

\section{Keywords:}

Molecular imprinting

Magnetic MIP

Langmuir and Freundlich adsorption isotherms Poly(2-pyrrolidone)

Polyamide 4

Bovine serum albumin

\begin{abstract}
A B S T R A C T
This study reports on the synthesis and adsorption properties of molecularly imprinted porous magnetic microparticles (MIP) based on the biodegradable and sustainable poly(2-pyrrolidone) (PPD or PA4). These new PPD MIP materials were obtained via activated anionic ring-opening polymerization of 2-pyrroldone carried out at $40{ }^{\circ} \mathrm{C}$, in the presence of iron fillers and bovine serum albumin (BSA) as a template. Neither solvent, nor additional crosslinking or porogen agents were used in the PPD MIP synthesis. Analogously, PPD particles without BSA imprinting (NIP) were also produced. Depending on the microparticles composition, their yields were in the 55-70 wt\% range, the average size varying between 8 and $25 \mu \mathrm{m}$. After characterization of the surface topography of all samples, their adsorption capacity toward the BSA target was assessed as a function of the adsorption time, protein concentration and $\mathrm{pH}$ of the medium. All three PPD MIP samples displayed adsorption capacity toward BSA being up to one order of magnitude higher as compared to other BSA-imprinted polymer systems. It was found that the rebinding of BSA on MIP is best described by the Langmuir isotherm, whereas for rebinding on NIP the Freundlich isotherm was the more adequate model. On this basis, the nature of the adsorption on MIP and NIP was discussed. The adsorption toward two other proteins, namely Ovalbumin and Cytochrome $C$ was also tested. The newly synthesized BSA-imprinted PPD MIP displayed selective adsorption for the BSA target being dependent on the $\mathrm{pH}$ values of the medium. The easy recovery of the Fe-containing MIP and the capacity of all MIP samples for multiple sorption/desorption cycles was demonstrated.
\end{abstract}

\section{Introduction}

The molecular imprinting technology (MIT) is a method for manipulation of the micro- and nanostructure of synthetic polymers creating in them tailor-made recognition sites that are complementary to a specific template: molecule, macromolecular assembly or even microorganisms [1]. A molecularly imprinted polymer (MIP) is formed by polymerization of a functional monomer around the template, typically in the presence of a cross-linker and a porogen [2]. Prior to the polymerization, the template and the functional monomer interact to form complexes, whose nature and exact constellation defines the architecture of the recognition sites and distinguishes the different types of MIT from each other [3]. The concluding stage of MIP formation comprises full or partial elimination of the template molecule being necessary for the recognition to occur in the spaces vacated by the imprinted species. The final MIP material contains a three-dimensional network presenting cavities with geometry and positions of the functional groups (i.e., a molecular lock) complementary to those of the template (a matching molecular key) [1-3].
Since the pioneering work of Polyakov in the 1930s using silica templates [4], there has been continuous development in the areas of design, preparation, characterization, and applications of MIPs. Nowadays there exist a vast number of scientific studies, many excellent reviews [1,5-8] and monographs [9] on this interdisciplinary subject. The elevated scientific interest resulted in gradual maturation of MIT opening many pathways for potential industrial application of MIP materials, e.g., in separation and purification of liquids and gases, in chemical and biochemical sensors, artificial antibodies, as a part of drug delivery systems and heterogeneous catalysis, among others [6]. Applications of MIP for selective recognition of explosives has also been reviewed recently [10].

There are two main types of MIP materials based on either covalent and noncovalent interactions between the template and the functional monomer. The covalent imprinting requires creation of chemical bonds between the template and the monomer and is generally considered more complex to realize [5]. Non-covalent imprinting can proceed by ionic bonding, van der Waals forces, $\pi-\pi$ interactions or hydrogen bond formation.

\footnotetext{
* Corresponding author.

E-mail address: denchev@dep.uminho.pt (Z.Z. Denchev).
} 
The role of the functional monomer in MIT is twofold: (i) to form a pre-polymerization complex with the template by providing appropriate functional groups and (ii) to produce the imprinted polymer in the form of micro- or nanoparticles, membranes, in-situ prepared monoliths or monolayers. The functional monomers most frequently used in the noncovalent interaction belong to the group of vinyl- and vinylidene monomers, such as acrylic or methacrylic acids and their polymerizable derivatives, styrene and its derivatives, vinyl pyridines, and similar [11]. The vast majority of MIPs for a wide range of functional groups and template structures are commonly prepared by some kind of free radical polymerization, initiated either thermally or photochemically [12-15]. The synthesis of MIP by atom-transfer radical polymerization (ATRP) [16] and click polymerization [17] was also reviewed. In most of the cases, the MIP polymerization should involve an appropriate solvent-porogen to create the necessary porous structure facilitating the template elution, as well as one or more bi-functional crosslinking monomers to fix the key-building block complexes during the 3D-polymer network formation $[2,18]$.

So far, MIPs have been successfully applied for the recognition and detection of a wide range of small organic moieties: metal ions such as $\mathrm{Pb}(\mathrm{II}), \mathrm{Hg}(\mathrm{II}), \mathrm{UO}_{2}{ }^{-2}$, and entire molecules of pesticides, fungicides, amino acids, antibiotics, among others [1]. Templates for MIPs representing large and finely, multi-level structured biological species for MIPs as peptides and proteins [19,20], viruses [21], and cells [22,23] have also been reported, however, successful imprinting in these cases still remains a great challenge $[8,24]$. The principal problem here is related to the quite complex and easily changing three-dimensional structure of these templates that could be denaturated or otherwise upset in the process of the MIP-forming polymerization [11,12]. Proteins are poorly soluble in organic solvents. They can additionally cause undesirable changes in their 3D structure during the MIP synthesis. Moreover, the large molecules of the protein template could remain arrested in the porous cross-linked MIP structure thus obstructing the template elution. To avoid this, a very precise control of the copolymerization of the crosslinking agent is required to ensure a complete template elution and sufficient analyte absorbance during rebinding [25].

Most biological templates such as proteins and enzymes are structural homologs of polyamides (nylons). This fact makes the latter quite attractive as MIP lock building blocks for imprinting of biomacromolecules. Thus, Dmitrienko et al. [26] reported the use of polyamide 6 (PA6) for the molecular imprinting of proteins, hemoglobin and adenosine triphosphate. Their method is based on precipitation of preformed PA6 from a water/fluorinated alcohol solution in the presence of DMFA porogen and the respective biomolecule. The advantage of this method as compared to others resulting in molecularly imprinted polyamides based on physical methods $[27,28]$ is that the MIP forms at $25{ }^{\circ} \mathrm{C}$. However, the use of highly aggressive organic solvents that are difficult to eliminate and the fact that the resulting PA6-based MIPs are not biodegradable are restrictive for many biotechnological applications.

Among the polyamide group, poly-2-pyrrolidone (PPD), i.e. polyamide 4 (PA4) is very attractive and environmentally promising polymer due to its biodegradability, biocompatibility and good mechanical performance, accompanied by a very high density of the amide hydrogen bonds. Unfortunately, the engineering applications of PA4 are limited by its low thermal resistance. However, this is not a drawback for biotechnology where the low-temperature processes are predominant. Therefore, PA4 can be an excellent candidate for lockbuilding blocks in protein imprinting. There is no information up to now about the use of activated anionic ring opening polymerization (AAROP) of lactams for the development of polyamide-based MIP. Very recently a new method was reported producing PPD microparticles in good yields, with controlled size, shape, and porosity by AAROP of 2pyrrolidone (2PD) [29]. The one-pot process was performed at temperatures as low as $40{ }^{\circ} \mathrm{C}$, can be applied for the preparation of magnetic-responsive microparticles and does not require solvents or other additional components.

The main objective of this work is to use low-temperature and solventless AAROP to synthesize smart protein-imprinted PPD-based microparticles with magnetic response using two types of Fe magnetic particles and bovine serum albumin (BSA) as a template. The kinetics of BSA rebinding was studied, followed by adsorption isotherms analysis and evaluation of their adsorption capacity as a function of the BSA concentration and $\mathrm{pH}$ of the medium. Selectivity studies with rebinding of other proteins were also performed. The reusability of the synthesized PPD-MIP systems was also assessed by multiple sorption/desorption experiments toward BSA target at three different $\mathrm{pH}$.

\section{Experimental}

\subsection{Materials}

The 2PD functional monomer, the template protein BSA (lyophilized powder) and all solvents used in this work are of analytical grade and were supplied by Sigma Aldrich - Portugal. As activator of the anionic polymerization the commercial product Brüggolen C20 from Brüggemann Chemical (Germany) was used that according to the manufacturer contains $80 \mathrm{wt} \%$ of aliphatic diisocyanate blocked in ECL. The polymerization initiator sodium dicaprolactamato-bis-(2-methoxyethoxo)-aluminate (Dilactamate, DL) is also a commercial product purchased from Katchem (Check Republic) and used without further treatment. Soft, non-insulated iron particles (Fe content $>99.8 \%$ ), with average diameters of 3-5 $\mu \mathrm{m}$ were kindly donated by the manufacturer BASF, Ludwigshafen, Germany. Two types of such iron particles were used - without (Fe) and with phosphate coating $(\mathrm{Fe}(\mathrm{C}))$. The ovalbumin (OVA, lyophilized powder, $>98 \%$ ) and the Cytochrome-C (Cyt-C, lyophilized powder, $>95 \%$ ) proteins used in the rebinding selectivity studies were supplied by Sigma Aldrich. All buffer solutions (0.1 M phosphate buffers with $\mathrm{pH} 4.0,5.0$ and 7.0) were prepared with double-distilled water.

\subsection{Synthesis of MIP and NIP}

The low-temperature AAROP of 2PD to PPD magnetic responsive microparticles was described in detail in a previous publication [29]. Fig. 1S of the Supporting Materials presents the reaction scheme. For the synthesis of magnetic responsive PPD-MIP the same process was applied occurring in the presence of BSA. Thus, $0.2 \mathrm{~mol}$ of $2 \mathrm{PD}$ were stirred with $1.0 \mathrm{wt} \%$ lyophilized BSA and $1 \mathrm{wt} \%$ of $\mathrm{Fe}$ or $\mathrm{Fe}(\mathrm{C}) \mathrm{mi}-$ croparticles at $25{ }^{\circ} \mathrm{C}$ for $30 \mathrm{~min}$. Subsequently, the $\mathrm{C} 20$ activator and the DL initiator were added under stirring, in inert atmosphere. Then, the temperature of the reaction mixture was set to $40{ }^{\circ} \mathrm{C}$ and the pressure to $50 \mathrm{mbar}$ for the next $7 \mathrm{~h}$. After AAROP completion, the resulting reaction mixture was dispersed in acetone and filtered thus removing most of the unreacted 2PD. To elute the BSA template, the raw PPD-MIP systems were dispersed consecutively in $1.0 \mathrm{M} \mathrm{NaCl}$, filtered, then washed with $0.1 \mathrm{M}$ borate buffer, $\mathrm{pH}=9.0$, filtered again and finally washed with distilled water. This procedure was repeated several times. After every cycle samples of the water filtrates were subjected to UV-spectroscopy. The elution of template protein from PPD-MIP samples was considered completed after the disappearance of the UV peak at $\lambda=286 \mathrm{~nm}$ typical of BSA. In order to eliminate the PPD oligomers, the resulting fine powders were extracted with methanol in a Soxhlet for $4 \mathrm{~h}$, dried in vacuum and stored in a desiccator. The respective samples without BSA imprinting (PPD-NIP) were prepared by the same procedure without introducing BSA into the polymerization mixture. The final PPD-NIP microparticles were washed subsequently with acetone and methanol and then subjected to a $4 \mathrm{~h}$ Soxhlet extraction with methanol. Table 1 shows the designation of all MIP and NIP samples synthesized in this study and some of their most important structural and morphological characteristics. 
Table 1

Sample designation and initial consolidated data of MIP and NIP samples.

\begin{tabular}{|c|c|c|c|c|c|c|}
\hline Sample designation & PPD Yield, $\%^{\mathrm{a}}$ & $\%$ of oligomers & Real Fe content, $\mathrm{R}_{\mathrm{L}}, \%^{\mathrm{b}}$ & $\eta, \mathrm{dL} / \mathrm{g}$ & $\mathrm{d}_{\max }, \mu \mathrm{m}$ & $\mathrm{d}_{\max } / \mathrm{d}_{\min }$ \\
\hline PPD-NIP & 59.4 & 4.4 & - & 0.923 & $8-15$ & $1.1-1.2$ \\
\hline PPD-MIP & 49.7 & 6.5 & - & 0.928 & $10-20$ & $1.2-1.3$ \\
\hline PPD-Fe-NIP & 69.9 & 3.5 & 2.10 & - & $10-20$ & $1.2-1.3 ;(3.4-4.0)$ \\
\hline PPD-Fe-MIP & 61.2 & 6.9 & $1.94^{\mathrm{c}}$ & - & $15-25$ & \\
\hline PPD-Fe(C)-NIP & 62.7 & 4.7 & 2.80 & - & $10-20$ & $1.2-1.3 ;(3.6-4.1)$ \\
\hline PPD-Fe(C)-MIP & 54.3 & 6.4 & $2.64^{c}$ & - & $15-25$ & \\
\hline
\end{tabular}

Notes: For magnetic responsive samples always $1 \mathrm{wt} \% \mathrm{Fe}$ or $\mathrm{Fe}(\mathrm{C})$ was used.

a In relation to the $2 \mathrm{PD}$ monomer.

b Determined by TGA, according to Eq. (1).

c Determined after elution of BSA from MIP.

\subsection{Structural characterization by spectral methods}

The PPD-MIP and PPD-NIP microparticles were subjected to Fourier-transform infra-red spectroscopy with attenuated total reflection (FTIR-ATR) in a Perkin-Elmer Spectrum 100 apparatus at a resolution of $2 \mathrm{~cm}^{-1}$ accumulating up to 16 spectra. In all of the protein adsorption and BSA elution experiments the respective concentrations were determined by UV-VIS spectroscopy using a Shimadzu UV2501PC spectrometer.

\subsection{Morphological, crystallization and thermal degradation studies}

The scanning electron microscopy (SEM) studies were performed in a NanoSEM-200 apparatus of FEI Nova using mixed secondary electron/back-scattered electron in-lens detection. The pulverulent samples were observed after sputter-coating with Au/Pd alloy in a $208 \mathrm{HR}$ equipment of Cressington Scientific Instruments with high-resolution thickness control. The real Fe load $R_{L}$ and the thermal stability of all MIP and NIP samples were established by means of thermogravimetric analysis (TGA) in a Q500 gravimetric balance (TA Instruments), heating the samples in the $40-600{ }^{\circ} \mathrm{C}$ at $20{ }^{\circ} \mathrm{C} / \mathrm{min}$ in a nitrogen atmosphere. The real Fe content, $R_{L}$, of the MIP and NIP was calculated according to Eq. (1):

$R_{L}=\left(R_{i}-R_{P P D}\right) \cdot 100,[\%]$

where $R_{P P D}$ is the carbonized residue at $600{ }^{\circ} \mathrm{C}$ of neat PPD particles and $R_{i}$ represents the carbonized residue of the respective metal-containing PPD-MIP or PPD-NIP sample.

\subsection{Adsorption kinetics}

Such studies assessing the adsorption capacity as a function of time were performed with both MIP and NIP systems to find out the time to reach the sorption-desorption equilibrium. Thus, $0.1 \mathrm{~g}$ of each powder sample were placed in a test tube containing $3.5 \mathrm{ml}$ of $1.0 \mathrm{wt} \%$ of BSA in $0.1 \mathrm{M}$ phosphate buffer ( $\mathrm{pH} 7.0)$. The total incubation time was $180 \mathrm{~min}$ at $37^{\circ} \mathrm{C}$, shaking the test tube at a constant rate. Aliquot amounts of $0.1 \mathrm{ml}$ were removed from the supernatant in the test tube after seven different incubation times in the 15-180 min range. These amounts diluted to $2 \mathrm{ml}$ with the same buffer were studied by UV spectroscopy determining the absorption of BSA at $\lambda=286 \mathrm{~nm}$. Using a calibration curve obtained with BSA standard, the concentration of protein in the supernatant after each incubation time was determined and from there - the amount adsorbed by the MIP/NIP system studied. Thereafter, the adsorption capacity $Q$ of the each PPD sample was determined [30]:

$Q=\frac{\left(C_{0}-C\right) V}{m}, \quad[\mathrm{mg} / \mathrm{g}]$

wherein $C_{0}[\mathrm{mg} / \mathrm{ml}]$ is the initial concentration of the BSA solution, $C$ $(\mathrm{mg} / \mathrm{ml})$ is the measured momentary concentration of BSA in the supernatant, $V(\mathrm{ml})$ is the volume of the BSA solution and $m[\mathrm{~g}]$ is the mass of the MIP or NIP sample. Relating the Q values of the MIP to that of the respective NIP sample, the imprinting factor IF was calculated [31]:

$I F=\frac{Q_{M I P}}{Q_{N I P}} \cdot 100[\%]$

\subsection{Adsorption isotherms}

These studies were carried out in a relatively broad concentration range and enabled to relate the adsorption process in all samples and conditions to an appropriate model. Thus, different initial BSA solutions were prepared with concentrations of $0.25,0.5,1.0,1.5$ and $2.0 \mathrm{wt} \%$, each concentration in $0.1 \mathrm{M}$ phosphate buffers with three $\mathrm{pH}$ values 4.0, 5.0 and 7.0, i.e., below, approximately at, and above the isoelectric point of BSA [31]. Having in mind that the sorption-desorption equilibrium of BSA on PPD MIP and NIP systems is established between 60 and $120 \mathrm{~min}$ of incubation at $37{ }^{\circ} \mathrm{C}$, the isotherms were constructed exactly in this time interval. The procedure for each one of the PPD-MIP and PPD-NIP systems for a given BSA concentration and given $\mathrm{pH}$ of the medium can be exemplified as follows. In a test tube with $0.1 \mathrm{~g}$ of the pulverulent sample were added $3.5 \mathrm{ml}$ of a $0.25 \mathrm{wt} \%$ solution of BSA in $0.1 \mathrm{M}$ phosphate buffer at $\mathrm{pH}=7$. The test tube is incubated at $37{ }^{\circ} \mathrm{C}$ under shaking removing aliquots of $0.1 \mathrm{ml}$ of the supernatant after 60 , 90 and $120 \mathrm{~min}$. Each one of these aliquots was diluted with $1.9 \mathrm{ml}$ of phosphate buffer $(0.1 \mathrm{M}, \mathrm{pH}=7)$. The three resulting solutions were studied by UV spectroscopy determining the intensity of the peak at $286 \mathrm{~nm}$. This procedure was repeated for all of the five BSA concentrations at three different $\mathrm{pH}$ values, for three incubation times: 60 , 90 and $120 \mathrm{~min}$. Three UV measurements were performed in each point to enable error evaluation.

\subsection{Adsorption selectivity studies}

To evaluate the recognition capability of PPD microparticles molecularly imprinted with BSA, these MIPs were used as adsorbents of two different proteins, namely ovalbumin (OVA, $\bar{M}_{w}=43 \mathrm{kDa}$, isoelectric point $\mathrm{p} I=4.7)$ and Cytochrome $C\left(\right.$ Cyt C, $\bar{M}_{w}=12.4 \mathrm{KDa}$ e $\left.\mathrm{p} I=10.2\right)$ selected as competitors for the BSA template $\left(\bar{M}_{w}=68 \mathrm{kDa}\right.$ e $\left.\mathrm{p} I=4.9\right)$. Solutions of the three proteins were prepared with equal concentrations of $0.1 \mathrm{wt} \%$ in three different $0.1 \mathrm{M}$ phosphate buffers with pH7.0, 5.0 and 4.0. For each of these studies $0.1 \mathrm{~g}$ of the three MIP samples were used as adsorbents applying a procedure similar to that of the adsorption isotherms. The only difference was that the MIP samples were incubated for three times, i.e., between 60,120 and180 min to make sure that the sorption/desorption equilibrium was reached for the OVA and Cyt C. Calibration curves were used for the calculation of the OVA and BSA residual concentrations after certain incubation time, on the basis of the intensity of the UV peak at $286 \mathrm{~nm}$, while in the case of Cyt $\mathrm{C}$ the peak at $\lambda=410 \mathrm{~nm}$ was used. 


\section{Results and discussion}

\subsection{Synthesis and characterization of PPD MIP and NIP microparticles}

The preparation of PPD porous microparticles without molecular imprinting via AAROP of 2PD [26] proved that the DL/C20 catalytic system provides successful solventless synthesis of PPD microparticles at temperatures as low as $40{ }^{\circ} \mathrm{C}$, the final yields of polymer reaching $52-63 \mathrm{wt} \%$. The presence of ferromagnetic microsized particles into the reaction mixture did not affect the kinetic of AAROP reaction resulting in production of PPD magnetic responsive microparticles. As expected, the NIP samples of this work obtained under the same conditions displayed quite similar yields (Table 1). Applying AAROP to obtain BSAimprinted PPD-MIP microparticles without and with magnetic payloads showed 43-54 wt\% final monomer conversion, the lower values being due to the more rigorous washing/purification procedure with various solvents and filtrations. Notably, the anionic polymerization in this case uses the excess of the 2PD as a solvent, at temperatures excluding thermal denaturation or disruption of its secondary or tertiary structure. An additional advantage of using AAROP for the MIP synthesis is that the chain propagation is accompanied by PPD crystallization that affixes the topology of the porous microparticles making redundant any crosslinking or pore-forming agents.

The intrinsic viscosity [ $\eta$ ] (see the Supporting information)) of PPDNIP and PPD-MIP samples was almost identical - 0.92-0.93 dL/g (Table 1). It can be therefore concluded that the presence of BSA in the AAROP reaction medium did not inactivate the DL/C20 catalytic system, the 2PD conversion to PPD and the molecular weight of the PPD-MIP particles being identical to those of the PPD-NIP controls. Moreover, the $[\eta]$ values of our NIP and MIP samples are similar to those of PPD microspheres obtained by a different method involving AAROP of 2PD [32].

The average size of the MIP particles based on optical microscopy with application of the respective image-processing software was found to be $10-25 \mu \mathrm{m}\left(d_{\max }\right.$ in Table 1, Fig. $2 S$ of Supporting Information) and was always larger than that of the respective NIP controls $(8-15 \mu \mathrm{m})$. The roundness of the major fraction of either PPD-NIP or PPD-MIP particles $\left(d_{\max } / d_{\min }\right)$ (Fig. 3S) was in the 1.1-1.3 range, i.e., it does not depend on the BSA presence. The Fe-containing particles displayed $3-5$ wt $\%$ of aggregates with aspect ratio of up to 4.0 most probably resulting from attraction between magnetized particles prior to their coating by PPD.

More details on the morphology of the PPD-NIP and PPD-MIP particles can be obtained by SEM (Fig. 1). The micrographs of the PPD-NIP (line 1) display spheroidal porous particles with sizes of the individual particles between 5 and $8 \mu \mathrm{m}$ (1a), the latter forming also aggregates with average sizes close to $20 \mu \mathrm{m}$ (1b). The MIP samples before (images $2 \mathrm{a}$ and $2 \mathrm{~b}$ ) and after washing ( $3 \mathrm{a}$ and $3 \mathrm{~b}$ ) also reveal aggregation to entities with average sizes of 20-25 $\mu \mathrm{m}$. At larger magnifications the PA4-NIP samples display porous, scaffold-like topology with visible pore diameters in the range of $70-120 \mathrm{~nm}$. At the same time, the micrographs of MIPs before (2c) and after washing (3c) display average pore sizes of 130-180 nm and above $200 \mathrm{~nm}$, respectively. Notably, the pores in the MIP before washing that still may contain physically adsorbed BSA on the surface of the particles, are smaller than those in the final MIP that should be free of template protein molecules. The surface cavities in the MIP in image $3 c$ display also a well-expressed and quite specific topography being distinct from all other samples in Fig. 1.

Fig. 2 compares the surface morphologies at larger magnifications of NIP and MIP samples carrying Fe- (images a-c) and $\mathrm{Fe}(\mathrm{C})$ payloads (d-f). It can be seen that in the respective final MIPs after washing of the template protein, the average sizes of the pores on the surface vary in the 130-200 nm and 120-170 nm, respectively. It seems also that the Fe magnetic particles of both types with average diameters of 4-5 $\mu \mathrm{m}$ are not located on the surface but in the core of the MIP particles. As seen from the EDX traces in Fig. $2 \mathrm{~d}$ and $\mathrm{f}$, the FeK $\alpha$ and FeK $\beta$ peaks only appear if the electron beam is directed into the pores (location Z2), rather than if it hits a non-porous spot of the MIP surface (location Z1).

The SEM images in Figs. 1 and 2 support the supposition that the removal of the template molecules by selective elution frees the recognition sites on the surface of the MIP particles, thus making better visible the imprinted cavities. It can be therefore hypothesized also that due to the porosity of the MIPs, the multiple washing procedures with organic and aqueous solutions will reach, dissolve and remove the BSA molecules from the particleś interior as well.

The FT-IR spectra of PPD-NIP sample and of the respective MIP at different stages of its preparation are presented in Fig. 3. All spectra display clear peaks for Amid I at $1633 \mathrm{~cm}^{-1}$ and Amid II at $1535 \mathrm{~cm}^{-1}$ with almost identical intensities. This is a clear indication for the fixation in both NIP and MIP samples of the trans-conformation of the $\mathrm{NH}-\mathrm{CO}$ group, being typical for polyamides, which means that even at a temperature as low as $40{ }^{\circ} \mathrm{C}$ polymerization can occur. In the PPD-NIP sample (curve 1) a weak peak at $1710 \mathrm{~cm}^{-1}$ is registered attributable to terminal $-\mathrm{COOH}$ functional groups. In the PPD-MIP sample (curve 3 ) a similar peak exists being shifted to higher wavenumbers.

All spectra in Fig. 3 display strong bands slightly above $3250 \mathrm{~cm}^{-1}$ typical of associated NH groups found in all semi-crystalline polyamides. In addition to this, the PPD-MIP spectrum before BSA elution (curve 2) shows peaks at 3545 and $3460 \mathrm{~cm}^{-1}$ of the symmetrical and asymmetrical vibrations of associated (i.e., $\mathrm{H}$-bonded to $\mathrm{C}=\mathrm{O}$ ) terminal $\mathrm{NH}_{2}$ groups. The latter cannot belong to the PPD polymer, since the accepted mechanism of lactam AAROP excludes the presence of such groups in the neat PPD [33]. Therefore, the presence of the peaks at 3545 and $3460 \mathrm{~cm}^{-1}$ in the unwashed PPD-MIP sample must be attributed to entrapped protein template. As seen from curve 3 in Fig. 3, in the final PPD-MIP sample after washing, the $\mathrm{NH}_{2}$ bands do not disappear completely. Even after the consecutive elution of the BSA by various solvents described in the Experimental part, some small but observable amounts of the template protein remain within the porous PPD-MIP particles. The reasons could be that either the solvents cannot reach and remove all BSA entrapped in the core of the MIP particles, or that some BSA molecules (or their fragments) became chemically bonded to the chain ends of the PPD forming during the AAROP due to chain transfer to the BSA during the AAROP.

The TGA studies of all NIP and MIP samples presented in Fig. 4 are in favor of the second hypothesis. Let us first consider the TGA traces generated on PPD samples without magnetic particles (Fig. 4a). The PPD-NIP sample (curve 1) represents a typical sigmoidal decomposition curve in which the $5 \%$ weight loss occurs at $202{ }^{\circ} \mathrm{C}$. In the PPD-MIP after template elution (curve 3 ) the same decomposition level is registered at $231{ }^{\circ} \mathrm{C}$, the whole TGA curve being shifted with ca. $30{ }^{\circ} \mathrm{C}$ to higher temperatures, i.e., the final MIP sample represents higher thermal resistance. Similar behavior was observed in PPD obtained by AAROP of 2PD with subsequent conversion of the terminal lactam rings into $-\mathrm{NH}_{2}$ groups [34].

The mass of carbonized residue at $600{ }^{\circ} \mathrm{C}$ for the PPD-NIP and PPDMIP after elution is $1.2 \mathrm{wt} \%$ and $2.0 \mathrm{wt} \%$ respectively, whereas with the PPD-MIP before elution (curve 2) this value is close to $6 \mathrm{wt} \%$. These data indicate that ca. $85 \%$ of the BSA template was removed by the elution procedures. The non-eluted BSA (or part of it) should be chemically bonded to the PPD in the MIP sample, otherwise a clear lowtemperature degradation step in curve 3 should have appeared right after $100{ }^{\circ} \mathrm{C}$, as observed in the MIP sample before elution of BSA (Fig. 4a, curve 2).

The $\mathrm{Fe}$ and $\mathrm{Fe}(\mathrm{C})$ bearing NIP and MIP samples (Fig. 4b and c) display a degradation behavior very similar to that of the PPD-NIP and PPD-MIP, hence the same chemical modification of the terminal groups of the PPD chains by BSA fragments can be suggested. The shift between the TGA curves of the NIP and final MIP samples (Fig. 4b and c, curves 1 and 3) reaches $43-45^{\circ} \mathrm{C}$. Comparing the carbonized residues at $600{ }^{\circ} \mathrm{C}$ of the PPD-NIP to that of the metal-containing MIP and NIP allows the determination of the real $\mathrm{Fe}$ and $\mathrm{Fe}(\mathrm{C})$ contents (Table 1). 


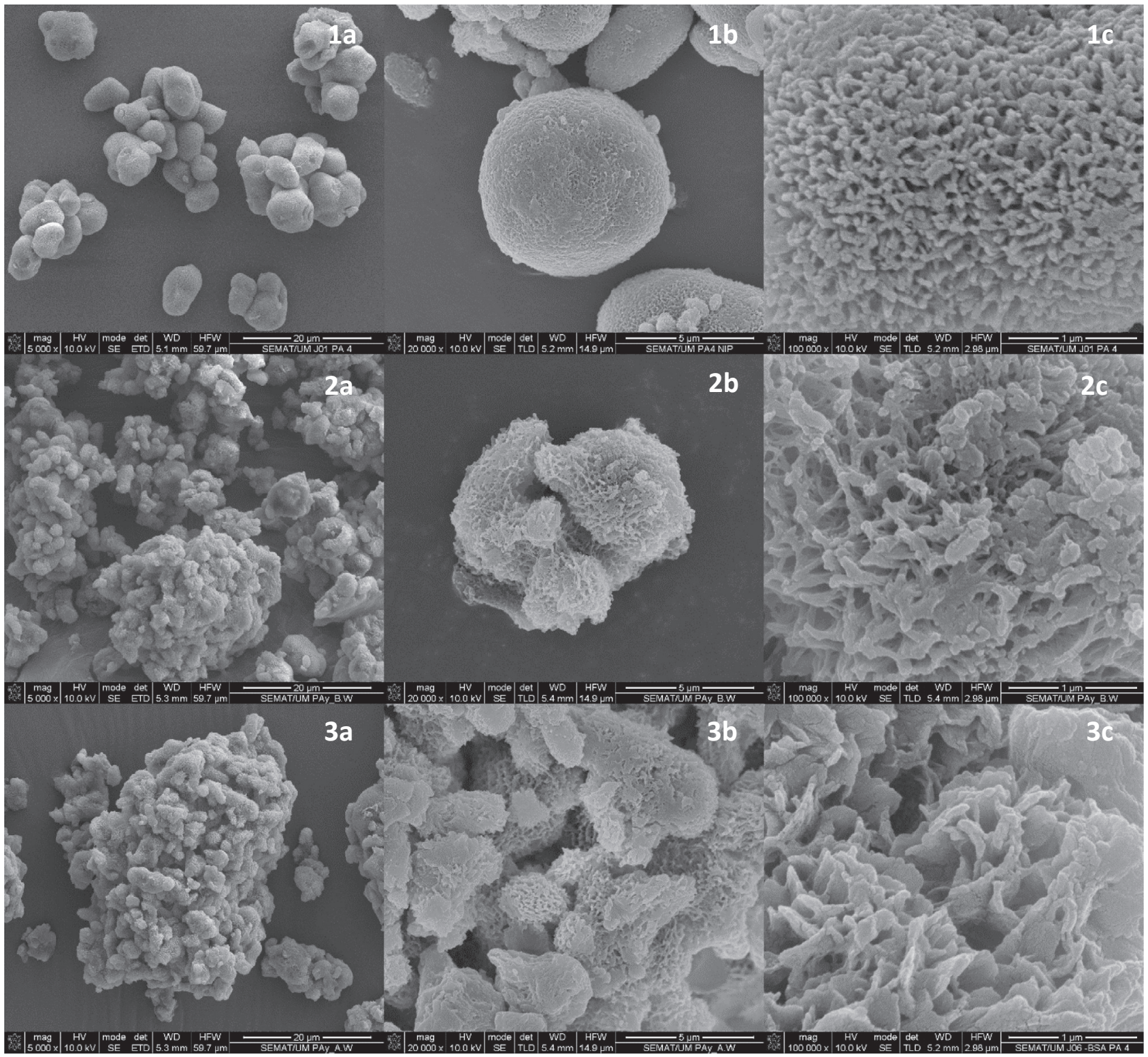

Fig. 1. Selected SEM images of PPD microparticles: 1 - PPD-NIP; 2 - PPD-MIP (before washing); 3 - PPD-MIP (after washing).

The latter varies between 2.0 and $2.5 \mathrm{wt} \%$, being dependent on the degree of conversion of $2 \mathrm{PD}$ to PPD.

\subsection{Rebinding properties of MIP and NIP with BSA}

\subsubsection{Kinetics of adsorption}

The first stage of studying the adsorption capacity $Q$ of the MIP/NIP sample pairs included a comparative investigation of its time dependence (Fig. 5, Table 2). This kinetic study was performed with all imprinted and not imprinted systems using $1.0 \mathrm{wt} \%$ of BSA in $0.1 \mathrm{M}$ phosphate buffer, $\mathrm{pH} 7$ and changing the adsorption time in the 0-180 min range.

Generally, the kinetic curves of MIP and NIP are similar in shape and display three zones. The initial fast linear increase of $Q$ between 0 and $20 \mathrm{~min}$ is followed by a loss of linearity of the curves between 20 and $60 \mathrm{~min}$, in some of the cases until $90 \mathrm{~min}$, then followed by a gradual stabilization after $90 \mathrm{~min}$.

Above 90 min all curves saturate indicating equilibrium in the adsorption/desorption process. Notably, during the initial $15 \mathrm{~min}$ of adsorption, the slopes of the curves for the PPD-Fe(C)-MIP/NIP (Fig. 5c) almost coincide displaying similar $Q$ values (Table 2), whereas the other two samples PPD-MIP (curve 5a) and PPD-Fe-MIP (curve 6b) displayed higher adsorption rate even in the beginning of the process.

With adsorption times larger than $20 \mathrm{~min}$, i.e., after the initial linear stage, the MIP samples display better binding capacity toward the BSA analyte. The differences between the $Q$-values of MIP and NIP reach at equilibrium values of $35-55 \mathrm{mg} / \mathrm{g}$ or $10-16 \%$ (Table 2 ). This is an indication of successful imprinting of the target protein in all of the three MIP samples of this study. It should be noted that their rebinding capacity toward the BSA target is from 5 to 6 times up to one of magnitude higher as compared to other BSA-imprinted MIP systems [16,35]. Furthermore, the proper NIP samples also display high values of $Q$, the main reason being most probably the similarity in the chemical structure of the BSA target and the PPD platform. Both contain amide functional groups that, along with the formation of H-bonds, are also capable of electrostatic interactions across the interface. The even higher adsorption capacity of the three PPD-MIP samples can be ascribed to the special recognition sites developed in MIP during the imprinting process. The kinetic study allowed also to find the necessary times for reaching the adsorption equilibrium, which was used 


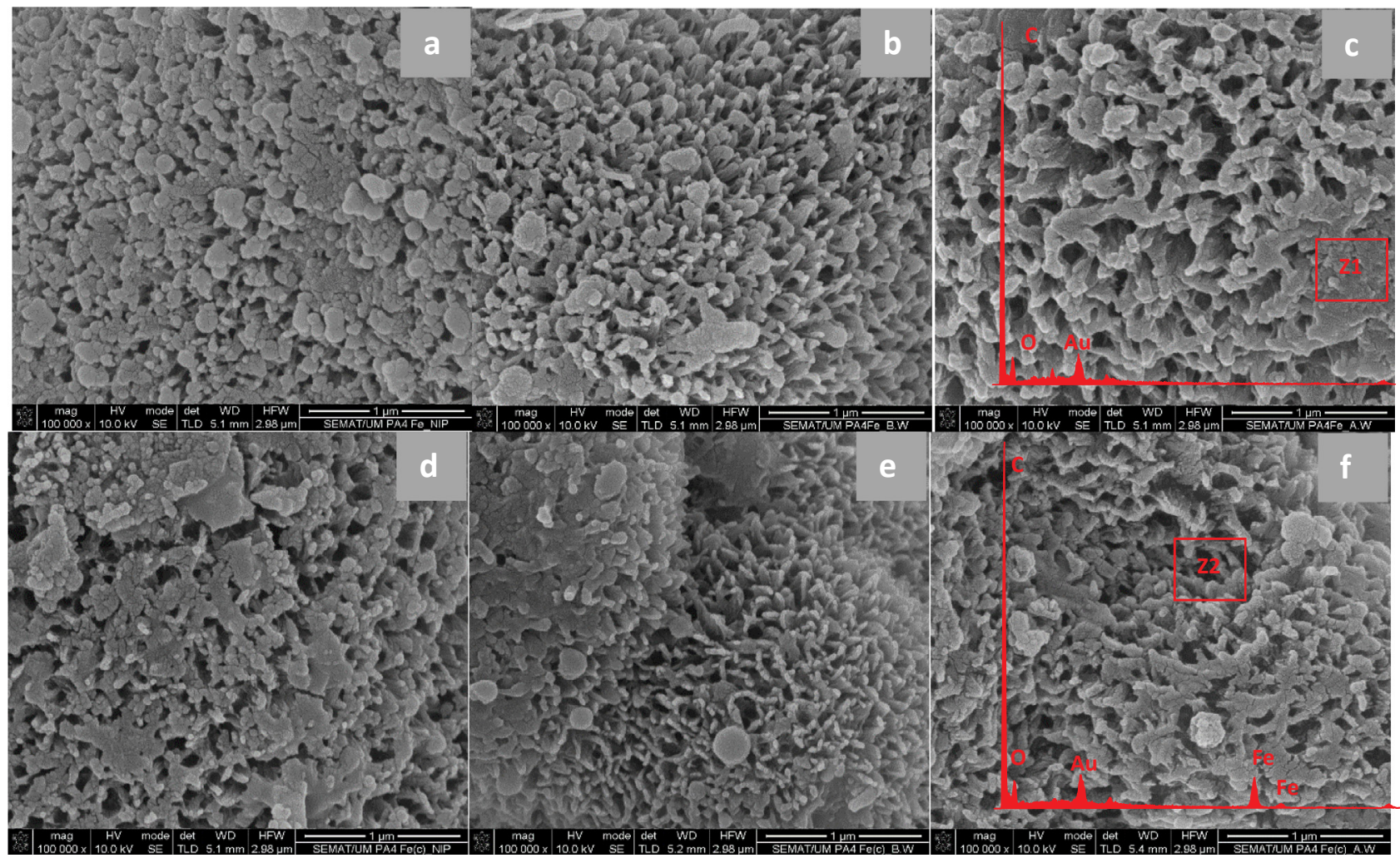

Fig. 2. Selected SEM images (maximal magnification) of $\mathrm{Fe}$ and $\mathrm{Fe}(\mathrm{C})$ containing PPD microparticles: a - PPD-Fe-NIP; b - PPD-Fe-MIP (before washing); $\mathrm{c}$ - PPD-FeMIP (after washing); d - PPD-Fe(C)-NIP; e - PPD-Fe(C)-MIP (before washing); f - PPD-Fe(C)-MIP (after washing).

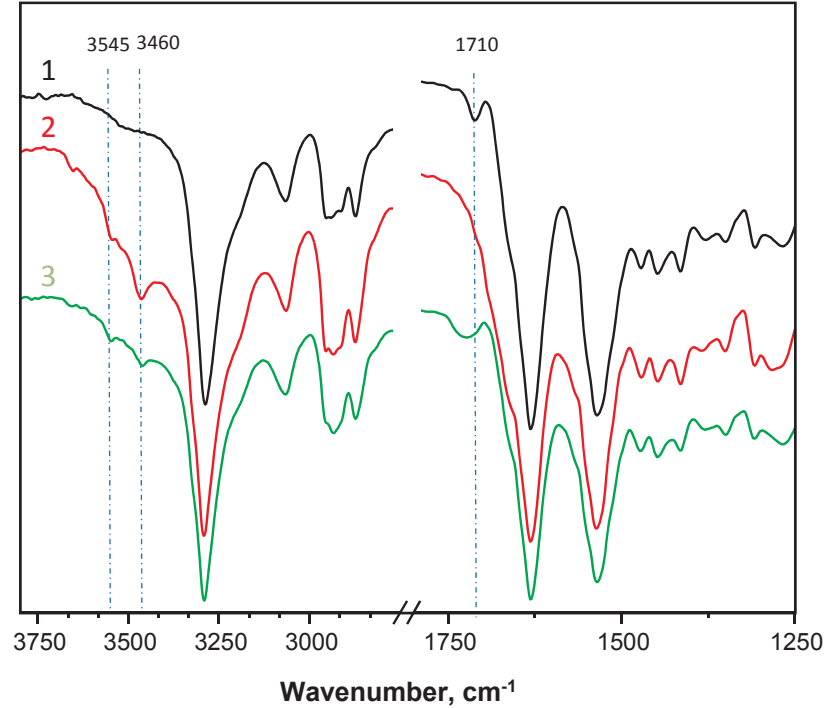

Fig. 3. Attenuated total reflection FT-IR spectra of PPD powders: 1 - PPD-NIP; 2 - PPD-MIP - before BSA elution; 3 - PPD-MIP - after BSA elution.

subsequently to assume the adequate adsorption model for each MIP system and conditions set.

\subsubsection{Absorption isotherms models}

To study further the nature of interactions between the MIP samples and the BSA template, the adsorption isotherms for all PPD-MIP samples were obtained at $37{ }^{\circ} \mathrm{C}$, using initial BSA concentrations in the $0.25-2.0 \mathrm{wt} \%$ range. Three different incubation times were applied, 60 , 90 and $120 \mathrm{~min}$. One more parameter that was varied was the acidity of the medium using starting BSA solutions in $0.1 \mathrm{M}$ phosphate buffers with pH7, 4 and 5, i.e., above, below and approximately at the isoelectric point of the BSA template. The consolidated results for all MIPs are presented in $Q_{e}=f\left(C_{e}\right)$ plots in Fig. 6 .

In each graph $C_{e}$ is the residual concentration of BSA in the solution at equilibrium $(\mathrm{mg} / \mathrm{ml})$ and $Q_{e}$ is the sample capacity of BSA absorption at equilibrium (mg BSA/g MIP). The visual inspection of the isotherms allows the conclusion that at $\mathrm{pH} 7$ and $\mathrm{pH} 5$ all of them are of the L-type, according to the classification of Gilles et al [36]. In this case, the $C_{e}$ and $Q_{e}$ values decrease with the increase of solute concentration indicating a progressive saturation of the MIP surface. At pH4, the Lcharacter of the curves is maintained for absorption times of 60 and $90 \mathrm{~min}$, while at $120 \mathrm{~min}$ no saturation of the $C_{e}$ e $Q_{e}$ values is registered, which indicates a different adsorption mechanism. One can hypothesize that under such conditions aggregation of BSA molecules could occur prior to their adsorption on the substrate. For shorter incubation times or lesser initial BSA concentration (e.g., below $2 \mathrm{wt} \%$ ) this effect is not observed, i.e., adsorption of single BSA molecules occurs.

To extract quantitative and qualitative information about the nature of the adsorption process, the isotherms in Fig. 6 should be interpreted in terms of a specific adsorption model. In this work, the isotherms were fitted to the models of Langmuir and Freundlich, which are the most frequently used for solid-liquid systems [37]. The theory of Langmuir deduces the following linearized relation between $C_{e}$ and $Q_{e}$ :

$\frac{C_{e}}{Q_{e}}=\frac{1}{Q_{\max } \cdot K_{l}}+\frac{1}{Q_{\max }} \cdot C_{e}$

where $Q_{\max }(\mathrm{mg} / \mathrm{g})$ is the theoretical maximum adsorption capacity of the MIP, and $K_{L}(\mathrm{~mL} / \mathrm{mg})$ is the Langmuir dissociation constant.

The linearized equation of Freundlich can be presented as:

$\log Q_{e}=\log K_{F}+\frac{1}{n} \log \cdot C_{e}$

where $K_{F}$ and $n$ are empirical constants characterizing a certain 

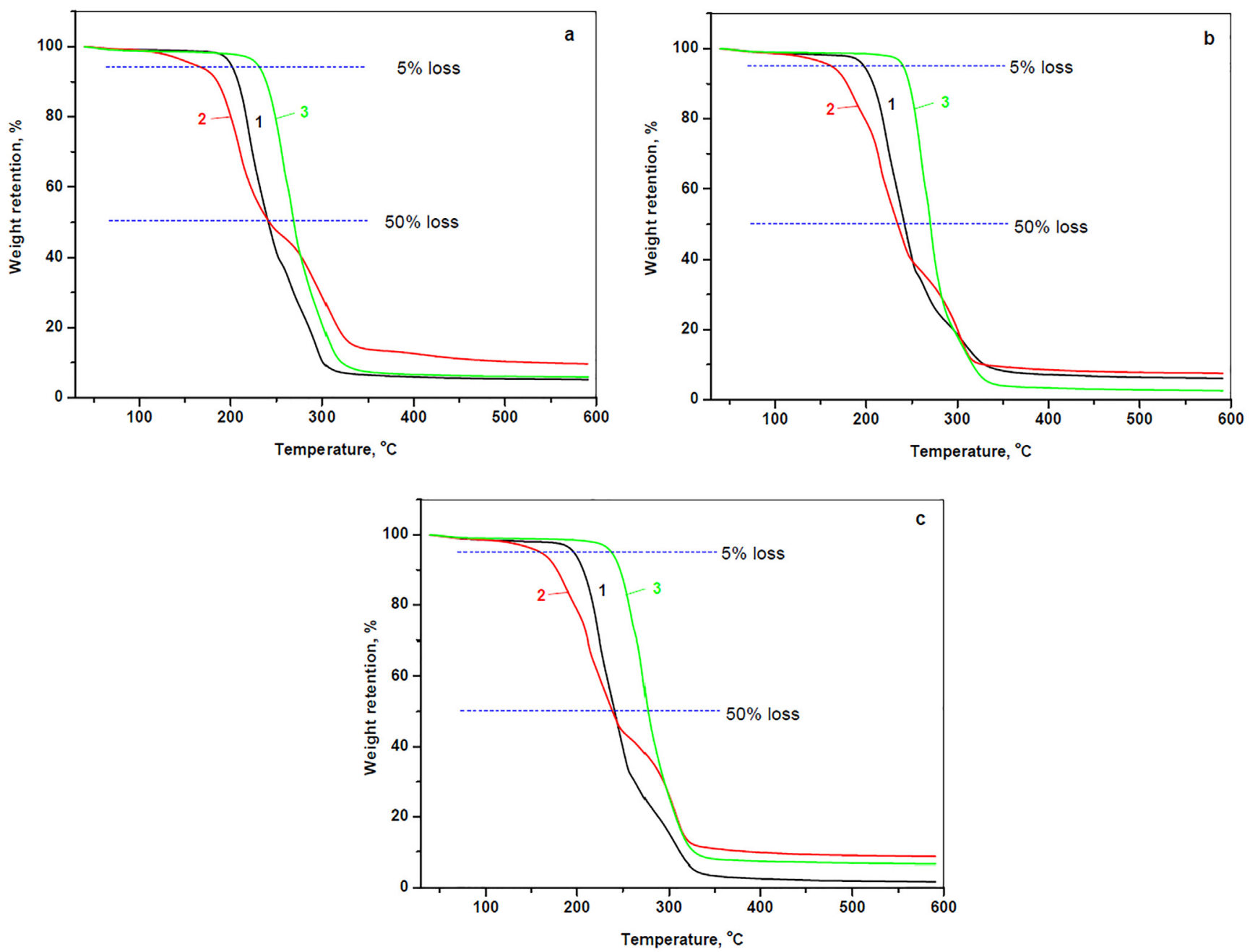

Fig. 4. TGA traces in the $40-600{ }^{\circ} \mathrm{C}$ range of: a - PPD; b - PPD-Fe; c - PPD-Fe(C). 1 - NIP; 2 - MIP before BSA extraction; 3 - MIP - after BSA extraction. For samples designation see Table 1.

adsorption process at a given temperature.

Thus, all curves in Fig. 6 were fitted to both Eqs. (4) and (5) using linear regression, calculating in the case of Langmuir model values for $Q_{\max }, K_{L}$ and the regression coefficient $R^{2}$. In the case of the Freundlich model, $K_{F}$ and $n$ were determined, along with the respective $R^{2}$ values. The fits of all isotherms related to MIP are presented in graphical form in Figs. 4S and 5S of the Supporting information. The respective numerical data are consolidated in Tables 3 and 4. The applicability of one or another model was decided after comparing the two regression coefficients for the same sample and condition, excluding the model with the lower $R^{2}$ value.

\subsubsection{Absorption isotherms analysis}

Comparing the $R^{2}$ values related to both adsorption models applied to all MIP samples allow the conclusion that for neutral and slightly acidic conditions (i.e., pH $5 \div 7$ ), it is the Langmuir model that describes the isotherms more adequately for all adsorption times. Having in mind the presuppositions of this model, under the said conditions, the BSA is expected to form monolayers upon the energetically homogeneous surface of the respective MIP adsorbent, without significant lateral interaction between the protein macromolecules. It should be noted that at $\mathrm{pH} 7$ the template protein is above its isoelectric point and hence bears a negative charge. At the same time, the MIP particles are also negatively charged with Z-potentials of $-31.5 \div-28.6 \mathrm{eV}$ (Table $1 S$ of the Supporting Information). The relatively high $Q_{\max }$ value of $450 \mathrm{mg} \mathrm{BSA} / \mathrm{g}$ MIP observed (Table 3, pH7) means that the efficiency of the adsorption process in this case should be attributed to the specific 3D surface architecture of the adsorbing PPD particles obtained during the imprinting. The presence of imprinted cavities exactly fitting the protein template molecules makes the non-covalent interactions between BSA monolayer and the MIP surface strong enough to overcome the expected electrostatic repulsion between the negatively charged protein macromolecules and MIP particles.

At $\mathrm{pH} 5$ the protein template is at its isoelectric point, i.e. electroneutral. At the same time, the Z-potential values of MIPs continue negative but are lower and vary between $-14.2 \mathrm{eV}$ for PPD-MIP and $-10.9 \mathrm{eV}$ for PPD-Fe-MIP. The excellent $Q_{\max }$ value of $>600 \mathrm{mg} / \mathrm{g}$ of the PPD-Fe-MIP after 60 min adsorption time shows the pure effect of the molecular imprinting of BSA not influenced negatively by electrostatic repulsion forces. Notably, under these conditions the PPD-Fe(C)MIP sample presents $Q_{\max }$ values of $556 \mathrm{mg} / \mathrm{g}$, whereas with the PPDMIP not bearing iron fillers $Q_{\max }$ drops to $357 \mathrm{mg} / \mathrm{g}$. Apparently, the presence of Fe filler can strongly influence the adsorption on MIP at certain $\mathrm{pH}$ values.

At pH4 and adsorption times of 60 min (for samples PPD-MIP and PPD-Fe(C)-MIP the interval is extended to $90 \mathrm{~min}$ ), the Langmuir model continues to be more adequate with $R^{2}$ being higher than 0.98 (Table 3). There it is the PPD-MIP that displays the outstanding $Q_{e}$ value of $667 \mathrm{mg} / \mathrm{g}$ (60 min).

One would expect intuitively that with the increase of the incubation time $Q_{\max }$ would either increase or saturate. For several samples in Table 3 (i.e., (PPD-MIP, pH 7; PPD-Fe-MIP pH 5 and PPD-Fe-MIP pH 4)) 

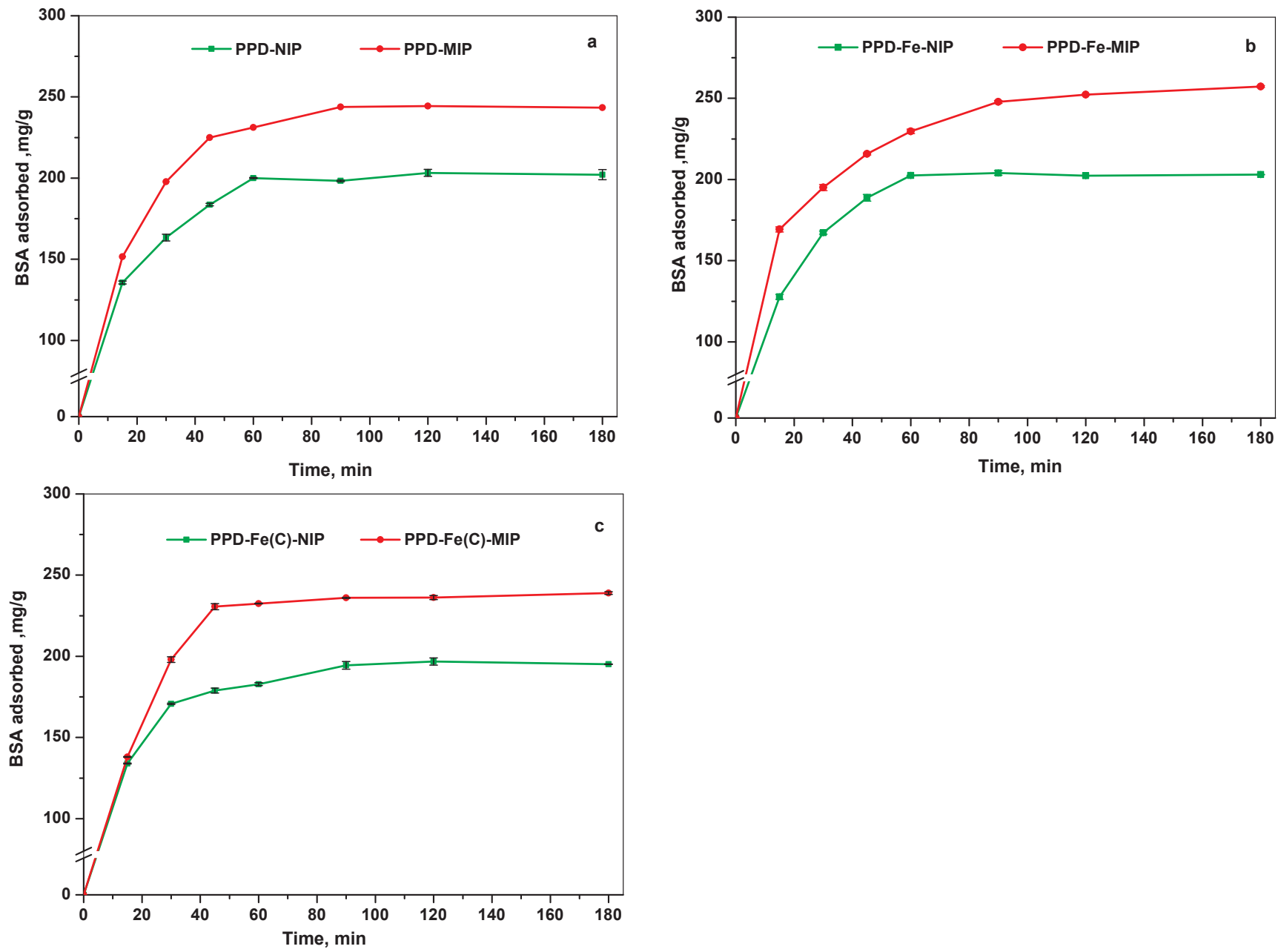

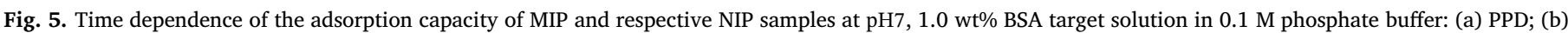
PPD-Fe; (c) PPD-Fe(C). The error bars are comparable with the point size.

Table 2

Absorption capacities in MIP and NIP samples after 15 min and after reaching the equilibrium of adsorption. The maximum possible adsorption capacity (total amount of BSA) is $350 \mathrm{mg} / \mathrm{g}$.

\begin{tabular}{lll}
\hline $\begin{array}{l}\text { Sample } \\
\text { designation }\end{array}$ & $\begin{array}{l}\text { Absorption capacity } Q \text { after } \\
15 \mathrm{~min}, \mathrm{mg} / \mathrm{g}^{\mathrm{a}},(\%)^{\mathrm{b}}\end{array}$ & $\begin{array}{l}\text { Absorption capacity } Q \text { at } \\
\text { equilibrium, } \mathrm{mg} / \mathrm{g}^{\mathrm{a}},(\%)^{\mathrm{b}}\end{array}$ \\
\hline PPD-MIP & $151.6 \pm 1.0(43.3 \pm 0.3)$ & $248.1 \pm 0.8(70.9 \pm 0.2)$ \\
PPD-NIP & $135.7 \pm 0.9(38.8 \pm 0.3)$ & $203.2 \pm 2.5(58.1 \pm 0.7)$ \\
PPD-Fe-MIP & $169.3 \pm 1.6(48.4 \pm 0.5)$ & $252.3 \pm 0.4(72.1 \pm 0.1)$ \\
PPD-Fe-NIP & $127.8 \pm 1.5(36.5 \pm 0.4)$ & $202.4 \pm 0.4(57.8 \pm 0.1)$ \\
PPD-Fe(C)-MIP & $138.1 \pm 0.2(39.5 \pm 0.1)$ & $238.1 \pm 1.2(68.0 \pm 0.3)$ \\
PPD-Fe(C-NIP & $133.9 \pm 0.3(38.3 \pm 0.1)$ & $196.7 \pm 2.2(56.2 \pm 0.6)$ \\
\hline
\end{tabular}

\footnotetext{
a As calculated according to Eq. (2).

b In respect to the total amount of BSA.
}

this is not the case, the $Q_{\max }$ values at 90 min being quite low. This observation may raise the question about a possible morphological inhomogeneity of the MIP samples that would affect the $Q_{\max }$ reproducibility. It should be noted that the MIP sample subjected to incubation times in the 60-120 min range is always the same with constant morphology or homogeneity. The adsorption capacity of the MIP particles changes because they start gradually to be covered by analyte molecules. It seems that with the MIP samples indicated after the initial "coating" with BSA for 60 min incubation, the equilibrium is shifted toward partial desorption. Whether or not this will happen and to what extend will probably depend on the topography of the MIP particles.
The latter, provided that the synthesis conditions were rigorously the same for all samples, will depend on the amount and type of magnetic particles implemented in MIP.

A notable exception from the Langmuir model is the adsorption time of $120 \mathrm{~min}$ (including also $90 \mathrm{~min}$ for the PPD-Fe-MIP), where the Freundlich model displays significantly better $R^{2}$ values for all MIPs (Table 4). In the latter case, one deals with adsorption upon energetically heterogeneous surfaces, i.e., formation of multilayers of BSA with strong interaction between the protein macromolecules. Moreover, $n$ values above 1 and close to 2 and the high $K_{F}$ values (Table 4, $\mathrm{pH}$ 4, $120 \mathrm{~min}$ ), obtained for all MIPs indicate quite favorable heterogeneous process with best adsorbate/adsorbent affinity.

A possible explanation of the preferential multilayer adsorption on MIPs at higher acidity and high adsorption times can be related to the electrostatic interactions between adsorbent and adsorbate. Thus, at pH 4 BSA is below its isoelectric point and therefore positively charged. As seen from Table 1S, the Z-potentials of PPD-MIP and PPD$\mathrm{Fe}(\mathrm{C})$-MIP are -3.8 and -2.7 , respectively, while the PPD-Fe-MIP has already a positive value slightly above 1 . Here, the non-covalent interactions due to the imprinted 3D structure of MIP will be complemented, especially after longer adsorption times, by the electrostatic attraction between BSA and MIP resulting in multilayer heterogeneous adsorption, in which the quantity of the adsorbed protein can infinitely grow. Table $1 \mathrm{~S}$ shows also that all NIP samples possess more negative Zpotential values as compared to the MIP counterparts. This effect can be explained with deprotonation and negative charges of the terminal $\mathrm{COOH}$ groups of the PPD macromolecules in NIP, while in MIP the 
PPD
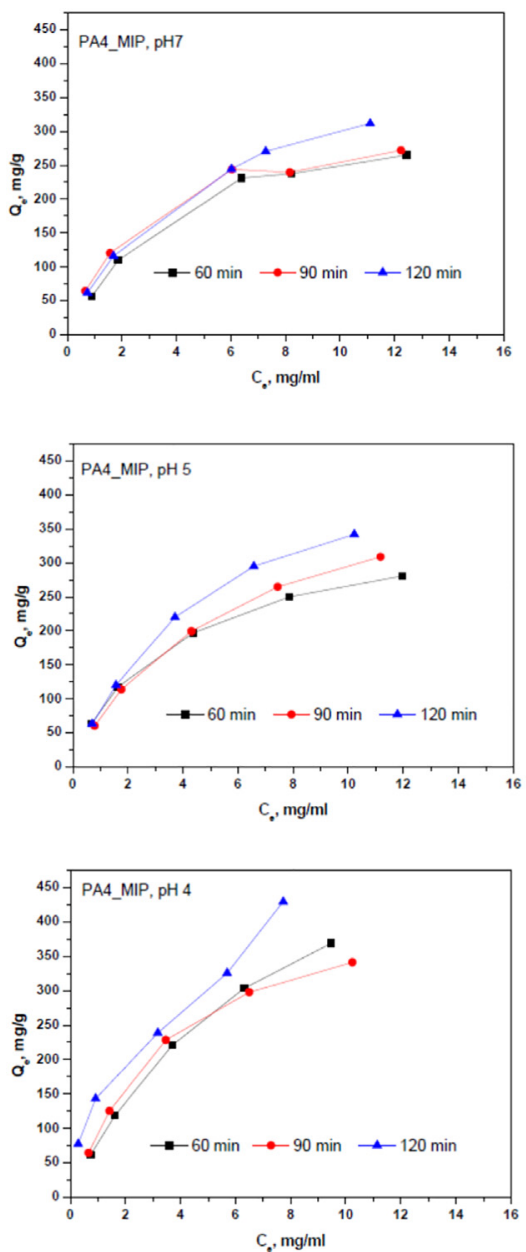

PPD-Fe
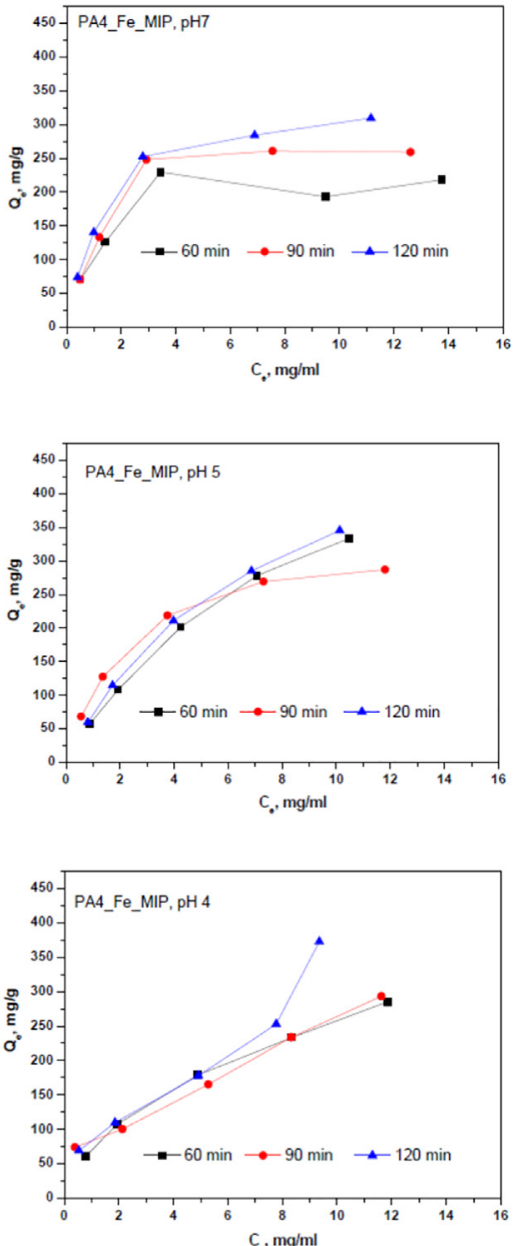

PPD-Fe(C)

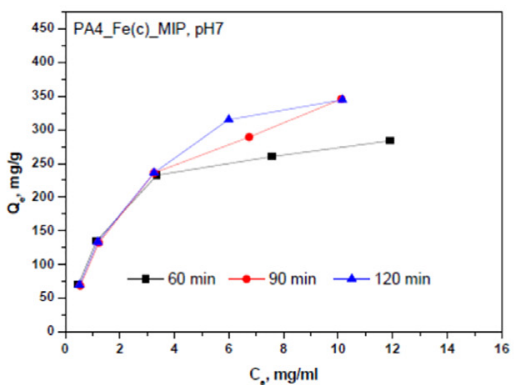

$\mathrm{pH} 7$

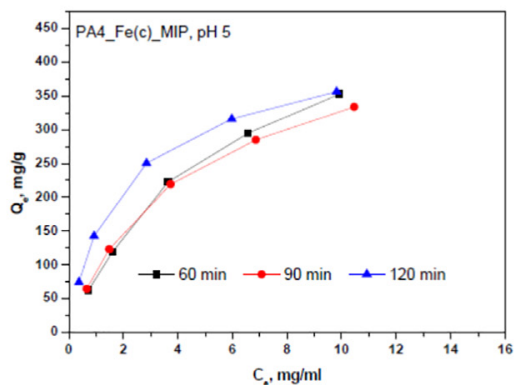

$\mathrm{pH} 5$

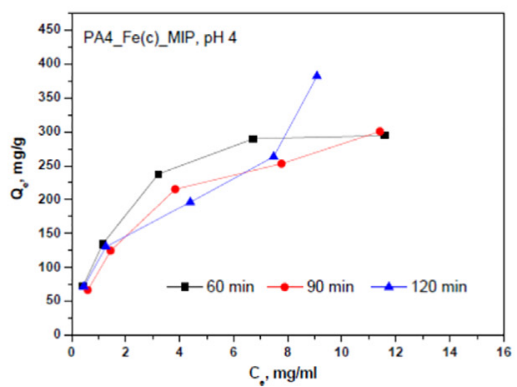

$\mathrm{pH} 4$

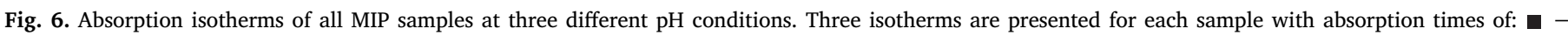
$60 \mathrm{~min}$; $-90 \mathrm{~min}$ and $\Delta-120 \mathrm{~min}$ - For samples designation see Table 1.

presence of $\mathrm{NH}_{2}$ groups at the terminuses that are prone to protonation and formation of $\mathrm{NH}_{3}{ }^{+}$moieties will decrease the negative charges of the particles.

Summarizing, the factors that determine the nature and the quantitative characteristics of BSA rebinding onto imprinted PPD samples are the $\mathrm{pH}$ of the medium, the time of adsorption and the presence of ferromagnetic particles in the MIP adsorbent. By appropriate control of these factors, one can achieve either adsorption in non-interacting monolayers governed by the recognition of the template molecules from the imprinted adsorbent, or non-controlled multilayer adsorption with strong interaction between the adsorbed layers. Under most of the conditions studied adsorption of single BSA molecules should be expected. It seems that at $\mathrm{pH}=4$ after long incubation and in a saturated BSA solution the analyte molecules could first aggregate and then adsorb on the substrate forming multilayers. Within the concentration range of $0.2-2.0 \mathrm{wt} \%$ of BSA, the isotherms of NIP are close to linear, not showing saturation. Evidently, NIPs can be used whenever adsorption of large amounts of protein is necessary on the same substrate.

Table 3

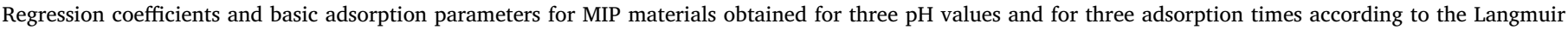
model. The bolded numbers represent the deviations from the Langmuir model.

\begin{tabular}{|c|c|c|c|c|c|c|c|c|c|c|}
\hline \multirow[b]{2}{*}{ Sample } & \multirow[b]{2}{*}{$\mathrm{pH}$} & \multicolumn{3}{|l|}{$R^{2}$} & \multicolumn{3}{|c|}{$Q_{\max }, \mathrm{mg} / \mathrm{g}$} & \multicolumn{3}{|c|}{$K_{L}, \mathrm{ml} / \mathrm{g}$} \\
\hline & & $60 \mathrm{~min}$ & $90 \mathrm{~min}$ & $120 \mathrm{~min}$ & $60 \mathrm{~min}$ & $90 \mathrm{~min}$ & $120 \mathrm{~min}$ & $60 \mathrm{~min}$ & $90 \mathrm{~min}$ & $120 \mathrm{~min}$ \\
\hline PPD-MIP & 7.0 & 0.987 & 0.993 & 0.999 & 368 & 329 & 441 & 0.223 & 0.380 & 0.215 \\
\hline PPD-Fe-MIP & & 0.982 & 0.982 & 0.998 & 228 & 291 & 357 & 0.11 & 0.81 & 0.72 \\
\hline PPD-Fe(C)-MIP & & 0.998 & 0.992 & 0.997 & 319 & 455 & 455 & 0.65 & 0.33 & 0.36 \\
\hline PPD-MIP & 5.0 & 0.999 & 0.999 & 0.999 & 357 & 455 & 513 & 0.30 & 0.19 & 0.20 \\
\hline PPD-Fe-MIP & & 0.996 & 0.999 & 0.999 & 613 & 444 & 585 & 0.12 & 0.45 & 0.14 \\
\hline PPD-Fe(C)-MIP & & 0.997 & 0.999 & 0.999 & 556 & 461 & 435 & 0.18 & 0.24 & 0.54 \\
\hline PPD-MIP & 4.0 & 0.999 & 0.998 & 0.887 & 667 & 500 & 526 & 0.14 & 0.24 & 0.40 \\
\hline PPD-Fe-MIP & & 0.969 & 0.791 & 0.594 & 388 & 358 & 500 & 0.20 & 0.25 & 0.17 \\
\hline PPD-Fe(C)-MIP & & 0.996 & 0.991 & 0.769 & 345 & 370 & 435 & 0.66 & 0.36 & 0.30 \\
\hline
\end{tabular}


Table 4

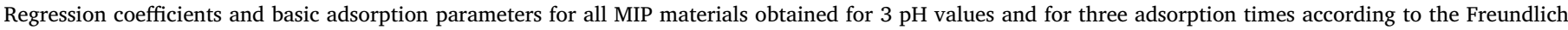
model.

\begin{tabular}{|c|c|c|c|c|c|c|c|c|c|c|}
\hline \multirow[b]{2}{*}{ Sample } & \multirow[b]{2}{*}{$\mathrm{pH}$} & \multicolumn{3}{|l|}{$R^{2}$} & \multicolumn{3}{|l|}{$n$} & \multicolumn{3}{|l|}{$K_{F}, \mathrm{ml} / \mathrm{g}$} \\
\hline & & $60 \mathrm{~min}$ & $90 \mathrm{~min}$ & $120 \mathrm{~min}$ & $60 \mathrm{~min}$ & $90 \mathrm{~min}$ & $120 \mathrm{~min}$ & $60 \mathrm{~min}$ & $90 \mathrm{~min}$ & $120 \mathrm{~min}$ \\
\hline PPD-MIP & 7.0 & 0.958 & 0.957 & 0.987 & 1.70 & 2.02 & 1.66 & 73.3 & 87.6 & 78.0 .9 \\
\hline PPD-Fe-MIP & & 0.719 & 0.801 & 0.898 & 3.00 & 2.50 & 2.36 & 106 & 116 & 129 \\
\hline PPD-Fe(C)-MIP & & 0.901 & 0.958 & 0.960 & 2.37 & 1.85 & 1.83 & 114 & 107 & 111 \\
\hline PPD-MIP & 5.0 & 0.976 & 0.983 & 0.979 & 1.93 & 1.61 & 1.56 & 84.4 & 74.8 & 85.7 \\
\hline PPD-Fe-MIP & & 0.991 & 0.948 & 0.987 & 1.38 & 2.10 & 1.44 & 65.7 & 10.1 & 74.3 \\
\hline PPD-Fe(C)-MIP & & 0.981 & 0.977 & 0.959 & 1.50 & 1.69 & 2.11 & 83.5 & 90.7 & 13.4 \\
\hline PPD-MIP & 4.0 & 0.983 & 0.963 & 0.990 & 1.37 & 1.64 & 2.03 & 77.6 & 93.5 & 14.5 \\
\hline PPD-Fe-MIP & & 0.998 & 0.986 & 0.922 & 1.77 & 2.48 & 1.83 & 71.5 & 93.8 & 87.4 \\
\hline PPD-Fe(C)-MIP & & 0.924 & 0.960 & 0.949 & 2.28 & 2.00 & 2.00 & 119 & 95.2 & 107 \\
\hline
\end{tabular}

As the process will produce multilayers, no selectivity should be expected in this case.

It should be noted also that no leaching of $\mathrm{Fe}^{2+}$ or $\mathrm{Fe}^{3+}$ ions was observed in the respective magnetic MIPs and NIPs (see the Supporting materials), which is an advantage for future biological applications.

\subsubsection{Comparison between MIP and NIP adsorption capacity}

Calculation of the $Q_{M I P} / Q_{N I P}$ relationship (i.e., the imprinting factor,

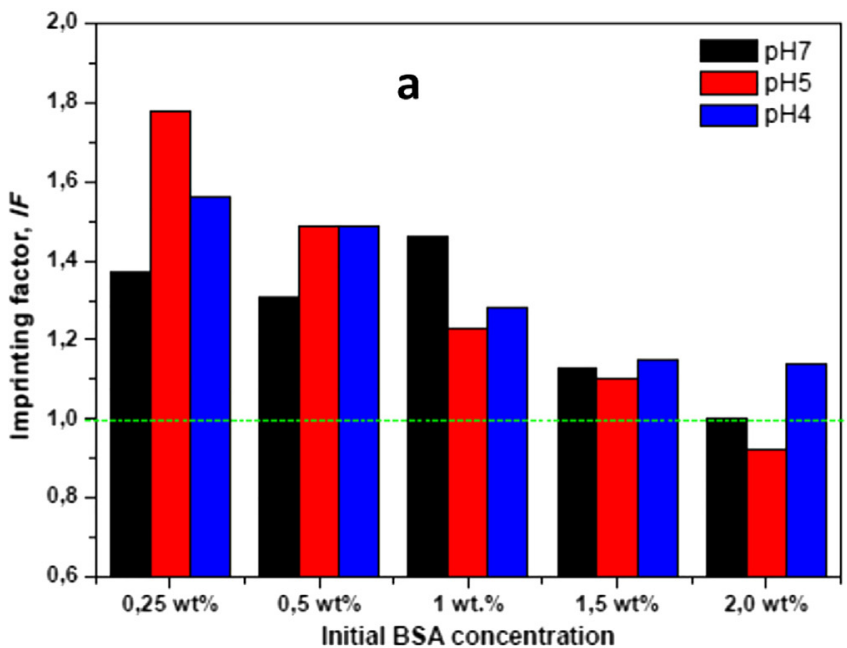

IF) as a function of the $\mathrm{pH}$ value and BSA concentration at a fixed adsorption time of $2 \mathrm{~h}$ is presented in Fig. 7. The IF values higher than 1 indicate better adsorption capacity of MIP, whereby at IF close to or lower than unity, the NIP particles show comparable or better adsorption capacity than MIP.

For all three samples the data clearly show that the IF depend not only on pH but also on the BSA initial concentration. At pH 5.0-7.0, i.e., at and above the BSA isoelectric point and initial concentration range of

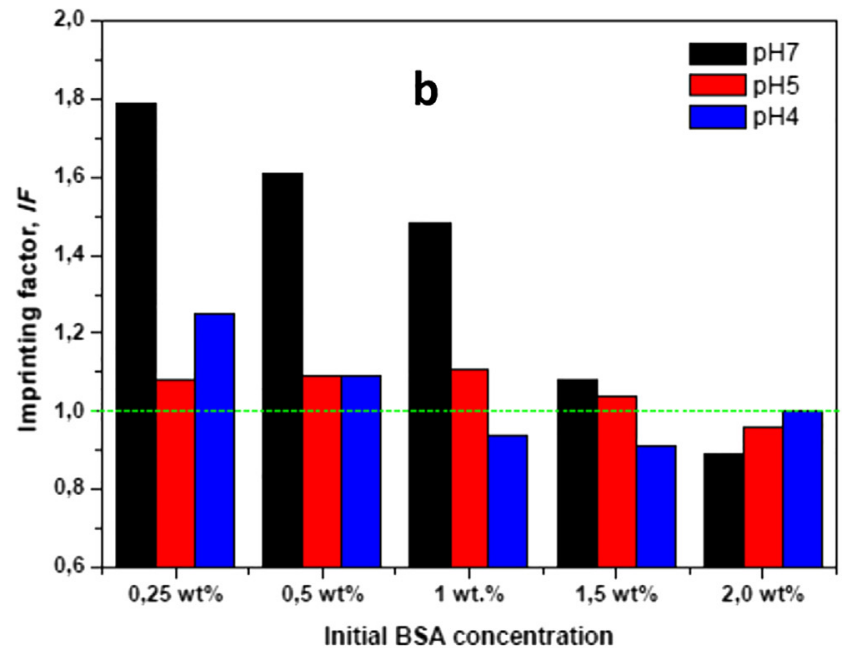

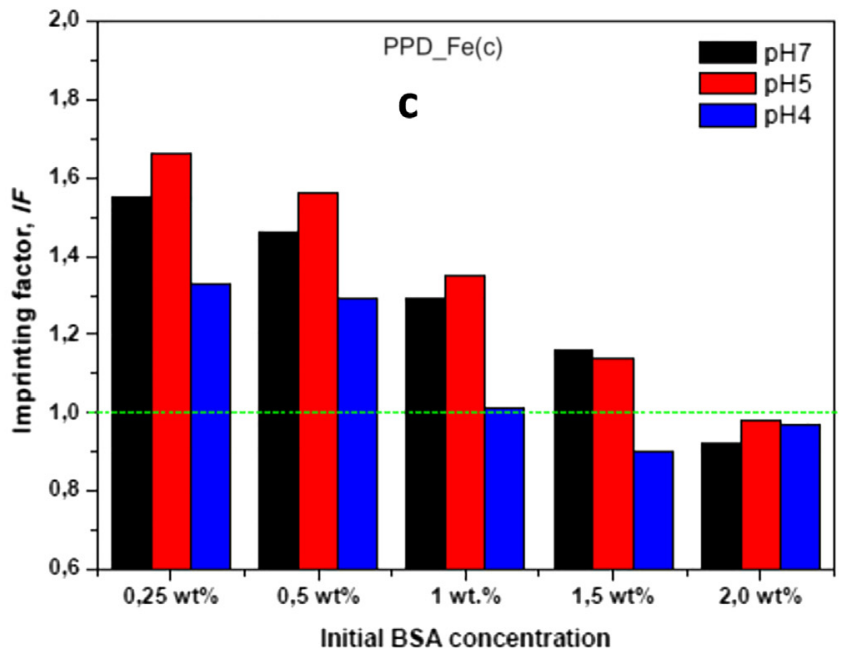

Fig. 7. Influence of the $\mathrm{pH}$ and the initial BSA concentration on the Imprinting factor IF $=\mathrm{Q}_{\mathrm{MIP}} / \mathrm{Q}_{\mathrm{NIP}}$, of the all three samples; a - PPD; $\mathrm{b}$ - PPD-Fe and c- PPD-Fe(C); $\mathrm{Q}_{\mathrm{MIP}}$ and $\mathrm{Q}_{\mathrm{NIP}}$ are determined after $2 \mathrm{~h}$ incubation time at $37{ }^{\circ} \mathrm{C}$. 
$0.25-1.0 \mathrm{wt} \%$ of BSA, the IF values are between 1.8 and 1.2 for the three systems - PPD, PPD-Fe and PPD-Fe(C). In this case, the adsorption obeys the Langmuir model presuming the formation of BSA monolayers upon a surface with a topography being complementary to the target protein. The PPD-Fe MIP (Fig. 7b) is definitely more effective than the respective NIP in neutral medium only, having maximum IF values between 1.5 and 1.8 at BSA solutions concentrations up to $1 \mathrm{wt} \%$. This system exemplifies in the best way the advantages of the molecular imprinting that governs the adsorption process in neutral medium. There, electrostatic attraction between PPD surface and target BSA protein BSA is excluded, even repulsion between equally charged target and MIP is to be expected. As regards the PPD-MIP and PPD-Fe(C)-MIP (Fig. 7a, c), these systems prove to be more useful in media with acidity close or below the BSA isoelectric point when BSA does not have charge, or both entities are oppositely charged.

Increasing the BSA concentration above $1.5 \mathrm{wt} \%$ irrespective of $\mathrm{pH}$ leads to IF values close or below 1 . At this condition it is the NIP particles that adsorb equally or even better than MIP. This should be related with the formation of multilayers of BSA related to the Freundlich adsorption model. There, the adsorption process depends predominantly on the interaction between these protein layers and less on the complementary surface topology created by the imprinting process.

\subsubsection{Rebinding selectivity of MIP with other proteins}

In order to evaluate the capacity of BSA-imprinted MIPs to recognize and adsorb other proteins, selectivity experiments were performed using, apart from the template BSA, two more target proteins: OVA, being with lower molecular weight and isoelectric point similar to that of BSA, and Cyt C with a significantly smaller molecule and much higher isoelectric point. All MIPs were incubated with $0.1 \mathrm{wt} \%$ initial protein concentration, at three different $\mathrm{pH}$, the adsorption time being 180 min (Fig. 8).

In a neutral medium, (Fig. 8a) the three MIP systems adsorb predominantly BSA (up to $85 \%$ of the initial concentration), whereby the adsorbed OVA amount is 2.5-5.0 times less. It should be noted that at pH7 both BSA and OVA are electronegative and will be repulsed electrostatically from the negatively charged MIP surface in a similar way. The big difference in the adsorption capability of BSA and OVA in this case, can be explained with the presence of cavities on the MIP surface with shape and active centers being complementary to the BSA template and not to OVA. It should be noted that the OVA macromolecule is smaller than that of BSA and therefore could easily be accommodated into the larger BSA-imprinted cavities. However, it seems that here the formation of recognition sites with proper geometry in the cavities is more important than the sizes of the latter. The adsorption capacity of Cyt $\mathrm{C}$ at neutral conditions as compared to BSA is with $60 \%$ inferior, that is one more notable experimental fact. At pH 7 Cyt C is electropositive and will be attracted to the MIP surface electrostatically. In addition, the molecule of this protein is much smaller which enhances additionally its adsorption. Still, it is the template BSA that displays better adsorption capacity, which should be attributed to the properly imprinted shell of the MIPs.

At pH 5 (Fig. 8b) the MIP samples show comparable adsorption capacities toward OVA and BSA (in both cases close to 85\%). Since the two proteins are at their isoelectric point, there are no possibilities for additional electrostatic interactions with the adsorbing surface of MIPs and the imprinted cavities accept almost equally BSA and OVA. Most probably, at $\mathrm{pH} 5$ the BSA and OVA have similar shape of their 3D structure. At the same time, Cyt C reaches only $c a$. $53 \%$ of its maximum adsorption capacity, even in the presence of attraction with the imprinted surface, i.e., the three MIP samples maintain their selectivity toward Cyt C.

At $\mathrm{pH} 4$ (Fig. 8c) the adsorption capacity of BSA template reaches its maximum (up to $92 \%$ on PPD-MIP), whereas the adsorption of OVA and Cyt C is 64\% and 54\%, respectively. At these conditions, the three proteins are below their isoelectric point, i.e. all are positively charged and capable of electrostatic interactions with MIP adsorbents. Nevertheless, the latter adsorb with preference the template protein. This means that below the isoelectric point of all proteins studied, the three PPD-MIPs retain their selectivity toward the BSA template. The increased adsorption capacity of the BSA-imprinted MIPs toward the template, as compared to the two other proteins in neutral and slightly acidic media justifies more systematic investigations on the adsorption selectivity of MIP.

\subsection{Recovery and reuse of PPD MIP}

In addition to the above structural and adsorptions investigations, it seems relevant to study two factors directly related to the practical application of the PPD-based MIP, namely their effective recovery from aqueous medium and reuse in multiple sorption/desorption cycles.

The introduction of ferromagnetic particles into MIP in the stage of AAROP was intended to enable MIP instant and complete recovery by means of simple magnetic attraction. Fig. 9 visualizes the separation and recovery of the BSA-loaded PPD-Fe-MIP sample. It should be noted that the PPD-Fe-MIP contains ca. $2 \mathrm{wt} \%$ of Fe which seems to be enough for an almost instant and complete separation from the initial water suspension.

The possibility to perform multiple protein adsorption/desorption cycles with the same PPD-based MIP is of great importance for its potential application as reusable matrix in biotechnological reactions. Fig. 10 displays the results of a 5-time adsorption/desorption of the BSA template studied for the three MIP systems of this work at three $\mathrm{pH}$ values.

The BSA adsorption was performed for $60 \mathrm{~min}$ from $1 \mathrm{wt} \%$ BSA solution as indicated in the Experimental part, Adsorption kinetics section. The BSA elution was performed according to the procedure for MIP purification. The results in Fig. 10 suggest that the MIP of this study can be reused five or more times, maintaining their adsorption capacity around $60 \%$ at $\mathrm{pH}=7$ and $\mathrm{pH}=5$. Notably, the Fe-charged MIP prepared for easier removal by magnetic interaction, perform, as a rule, slightly better than the neat PPD-MIP reaching higher BSA adsorption and equal or better removal during each cycle. It can be therefore concluded that the PA4-based MIP systems of this study have potential as reusable selective adsorbents for biotechnological applications.

\section{Conclusions}

A novel synthetic route was developed for molecular imprinting of bovine serum albumin template protein in porous PA4 microparticles that carry ferromagnetic fillers. It is based on activated anionic polymerization of 2-pyrrolidone ( $\gamma$-butyrolactam) carried out at $40{ }^{\circ} \mathrm{C}$ in the presence of the template protein and the Fe fillers. The combination of protein imprinting with magnetic susceptibility brings two advantages: selective rebinding of the target protein and easy collection by an external magnetic field. The process produces poly(2-pyrrolidone) microparticles with controllable porosity (100-200 nm) and average size of 15-25 $\mu \mathrm{m}$, in which BSA is entrapped. The major part of the entrapped BSA (ca. 85\%) can be eluted by washing with organic and aqueous solvents to produce the final molecularly imprinted PPD microparticles. The rest of the BSA or its fragments becomes chemically bonded to the terminuses of the PPD macromolecules. Whenever the $\mathrm{pH}$ of the BSA solution is neutral or equal to its isoelectric point and the BSA concentration does not exceed $1.5 \mathrm{wt} \%$, the adsorption capacity of the BSA-imprinted samples toward the template is with $50-80 \%$ higher than that of similar microparticles without imprinting. At these conditions the adsorption is described by the Langmuir isotherm, supposing the formation of monomolecular layers of BSA, non-covalently bonded to the imprinted PPD particle. The surface topology of the latter is complementary to the shape and active centers of the BSA macromolecules. Excellent maximal adsorption capacities of above $600 \mathrm{mg}$ 

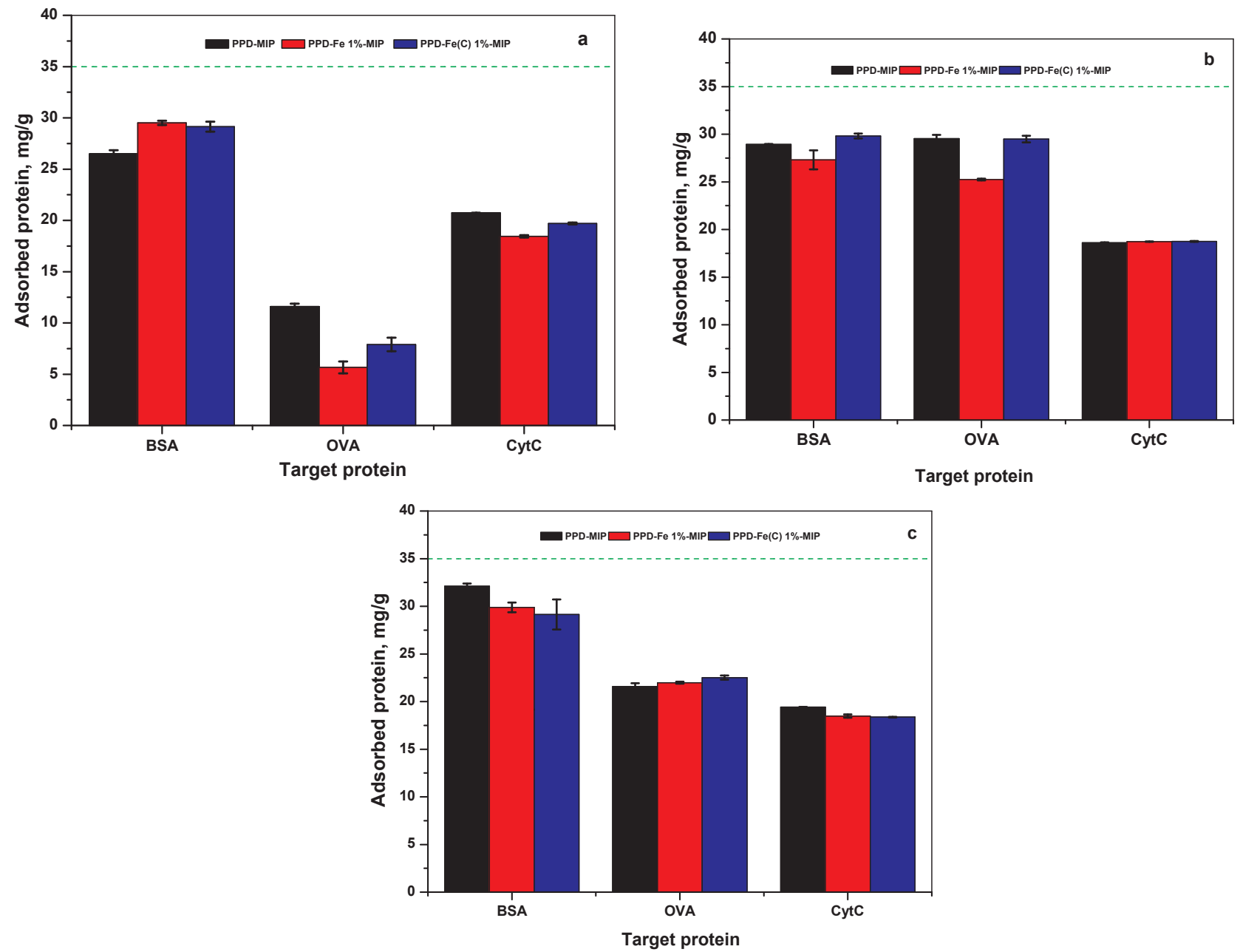

Fig. 8. Adsorption capacity of template BSA and other proteins (OVA and Cty C) on BSA-imprinted MIPs: a - pH 7.0; $\mathrm{b}-\mathrm{pH}$ 5.0; $\mathrm{c}-\mathrm{pH} 4.0$. The dashed lines indicate the maximum possible adsorption level.

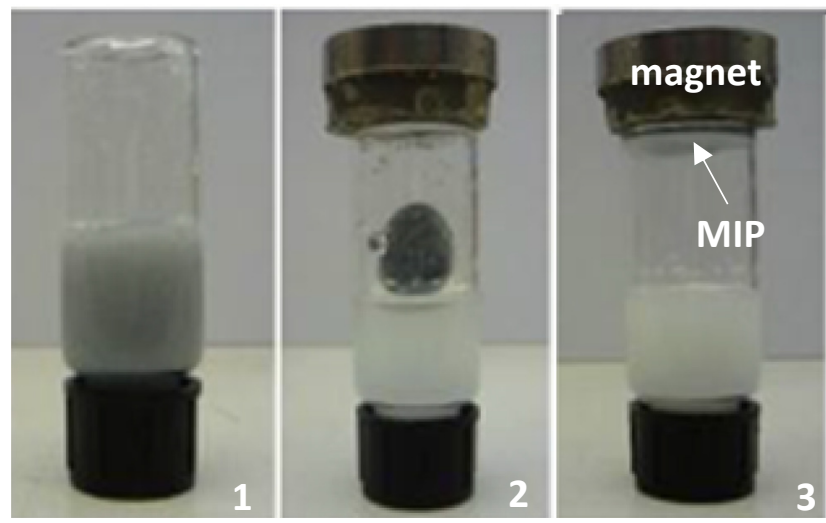

Fig. 9. PPD-Fe-MIP sample with adsorbed BSA: 1 - MIP suspension in water; 2,3 - phases of MIP separation applying a constant magnet.

BSA/g MIP were obtained at $\mathrm{pH}$ 5-7, being significantly higher than in the known BSA-imprinted MIP. At solution concentrations above $1.5 \mathrm{wt}$ $\%$ of BSA and $\mathrm{pH} 4$, the adsorption process with PPD MIP particles is more adequately described by the Freundlich isotherm. The BSA adsorption by all NIP samples at all concentrations and $\mathrm{pH}$ values studied is also described by the Freundlich model postulating multilayer adsorption with strong interaction between the protein layers. Under these conditions, the adsorption is not governed by the surface topography of the PPD particles. The new PPD-MIP systems display selectivity toward the template protein depending on the $\mathrm{pH}$ of the medium. The Fe-containing PPD-MIP can be easily and completely removed from water suspensions by simple magnetic interaction. All PPDMIP are reusable in five or more sorption/desorption cycles, whereby the more complete the desorption is carried out, the higher the new BSA load during the next cycle could be.

\section{Declaration of Competing Interest}

The authors declare that they have no known competing financial interests or personal relationships that could have appeared to influence the work reported in this paper.

\section{Acknowledgements}

The authors gratefully acknowledge the financial support of the project TSSiPRO NORTE-01-0145-FEDER-000015, supported by the regional operation program NORTE2020, under the Portugal 2020 Partnership Agreement, through the European Regional Development Fund, as well as the support by National Funds through Fundação para a Ciência e Tecnologia (FCT), project UID/CTM/50025/2019. N. Dencheva is also grateful for the financial support of FCT in the frames of the strategic project UID/CTM/50025/2013 and the personal program-contract CTTI-51/18-IPC. 

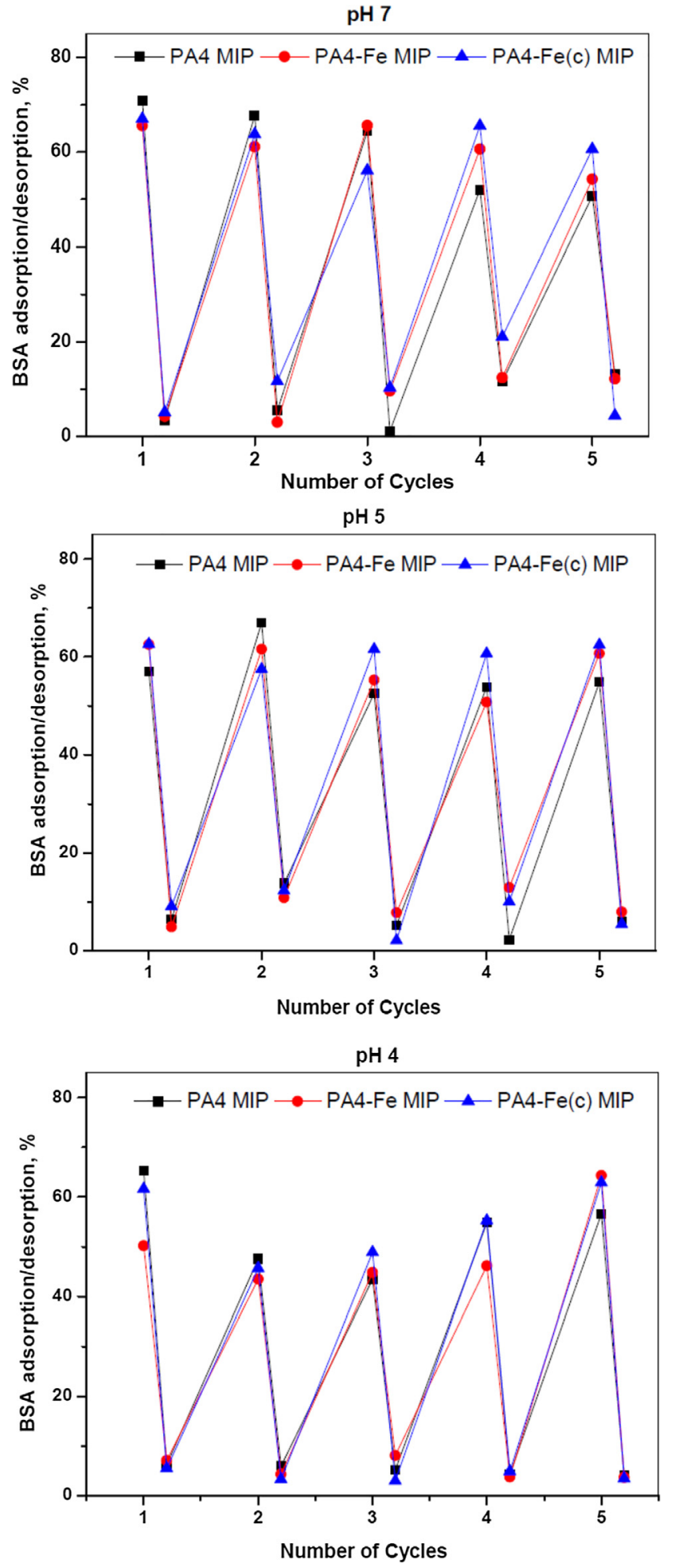

Fig. 10. Multiple adsorption/desorption in MIP samples at three different $\mathrm{pH}$ values.

\section{Appendix A. Supplementary material}

Supplementary data to this article can be found online at https:// doi.org/10.1016/j.eurpolymj.2019.109375.

\section{References}

[1] L. Chen, X. Wang, W. Lu, X. Wu, J. Li, Molecular imprinting - perspective and applications, Chem. Soc. Rev. 45 (2016) 2137-2211.

[2] H. Yan, K.H. Row, Characteristic and synthetic approach of molecularly imprinted polymer, Int. J. Mol. Sci. 7 (2006) 155-178.

[3] J.E. Lofgreen, G.A. Ozin, Controlling morphology and porosity to improve performance of molecularly imprinted sol-gel Silica, Chem. Soc. Rev. 43 (2014) 911-933.

[4] M.V. Polyakov, Adsorption properties and structure of silica gel, Zh. Fiz. Khim. Acad. SSSR 2 (1931) 799-805.

[5] C. Alexander, H.S. Andersson, L.I. Andersson, R.J. Ansell, N. Kirsch, I.A. Nicholls, J. O'Mahony, M.J. Whitcombe, Molecular imprinting science and technology: A survey of the literature for the years up to and including 2003, J. Mol. Recognit. 19 (2006) 106-180.

[6] L. Chen, L., S. Xu, J. Li,, Recent advances in molecular imprinting technology: current status, challenges and highlighted applications, Chem. Soc. Rev. 40 (2011) 2922-2942.

[7] R. Schirhagl, Bioapplications for molecularly imprinted polymers, Anal. Chem. 86 (2013) 250-261.

[8] S. Li, S. Cao, M.J. Whitcombe, A. Piletsky, Size matters: challenges in imprinting macromolecules, Prog. Polym. Sci. 39 (2014) 145-163.

[9] K. Haupt, A.V. Linares, M. Bompart, B. Tse Sum Bui, in: K. Haupt (Ed.), Molecular Imprinting, Springer, 2012, pp. 1-28.

[10] M. Zarejousheghani, W. Lorenz, P. Vanninen, T. Alizadeh, M. Cämmerer, H. Borsdorf, Molecularly imprinted polymer materials as selective recognition sorbents for explosives: a review, Polymers 11 (2019) 888, https://doi.org/10 3390/polym11050888.

[11] P.A.G. Cormack, A.Z. Elorza, Molecularly imprinted polymers: synthesis and Characterization, J. Chromatogr. B 804 (2004) 173-182.

[12] L. Qian, X. Hu, P. Guan, D. Wang, J. Li, C. Du, R. Song, C. Wang, W. Song, The effective specific recognition of bovine serum albumin imprinted silica nanoparticles by utilizing a macromolecular functional monomer to stabilize and imprint template, Anal. Chim. Acta (2015) 97-105.

[13] Y.W. Tong, C.J. Tan, Preparation of superparamagnetic ribonuclease A surfaceimprinted submicrometer particles for protein recognition in aqueous media, Anal. Chem. 79 (2007) 299-306.

[14] C.J. Tan, H.G. Chua, K.H. Ker, Y.W. Tong, Preparation of bovine serum albumin surface-Iimprinted submicrometer particles with magnetic susceptibility through core-shell miniemulsion polymerization, Anal. Chem. 80 (2008) 683-692.

[15] A. Navarro, A.L. Castillo, J.F. Sanchez, A. Gutierrez, Synthesis of a novel polyurethane-based magnetic imprinted polymer for the selective optical detection of 1 naphthylamine in drinking water, Biosens. Bioelectron. 26 (2011) 4520-4525.

[16] Q.-Q. Gai, F. Qu, T. Zhang, Y.K. Zhang, Preparation of bovine serum albumin surface-imprinted superparamagnetic polymer with the assistance of basic functional monomer and its application for protein separation, J. Chromatogr. A 1218 (2011) 3489-3495.

[17] X. Wang, Y. Pei, Y. Hou, Z. Pei, Fabrication of core-shell magnetic molecularly imprinted nanospheres towards hypericin via click polymerization, Polymers 313 (2019) 11, https://doi.org/10.3390/polym11020313.

[18] G.M. Gladis, T.P. Rao, Effect of porogen type on the synthesis of uranium ion imprinted polymer materials for the preconcentration/separation of traces of uranium, Microchim. Acta 146 (2004) 251-258.

[19] D. Cai, L. Ren, H. Zhao, C. Xu, L. Zhang, Y. Yu, H. Wang, Y. Lan, M.F. Roberts, J.H. Chuang, A Molecular-imprint nanosensor for ultrasensitive detection of proteins, Nat. Nanotechnol. 5 (2010) 597-601.

[20] Y. Hoshino, T. Kodama, Y. Okahata, K.J. Shea, Peptide imprinted polymer nano particles: a plastic antibody, J. Am. Chem. Soc. 130 (2008) 15242-15243.

[21] M. Gast, H. Sobek, B. Mizaikoff, Advances in imprinting strategies for selective virus recognition - a review, Trends Anal. Chem. 114 (2019) 218-232.

[22] Z. Zhang, M. Li, J. Ren, X. Qu, Cell-imprinted antimicrobial bionanomaterials with tolerable toxic side effects, Small 11 (2015) 1258-1264.

[23] O. Hayden, F.L. Dickert, Selective microorganism detection with cell surface imprinted polymers, Adv. Mater. 13 (2001) 1480-1483.

[24] W. Zhang, X.W. He, Y. Chen, W.Y. Li, Y.K. Zhang, Composite of CdTe quantum dots and molecularly imprinted polymer as a sensing material for cytochrome C, Biosens Bioelectron. 26 (2011) 2553-2558.

[25] N.W. Turner, C.W. Jeans, C.K.R. Brain, C.J. Allender, V. Hlady, D.W. Britt, From 3D to 2D: a review of the molecular imprinting of proteins, Biotec. Progress 22 (2006) 1474-1489.

[26] E.V. Dmitrienko, R.D. Bulushev, K. Haupt, S.S. Kosolobov, A.V. Latyshev, I.A. Pyshnaya, D.V. Pyshnyi, A simple approach to prepare molecularly imprinted polymers from Nylon-6, J. Mol. Recog. 26 (2013) 368-375.

[27] P.S. Reddy, T. Kobayashi, N. Fujii, Molecular imprinting in hydrogen bonding networks of polyamide nylon for recognition of amino acids, Chem. Lett. 28 (1999) 293-294.

[28] P.S. Reddy, T. Kobayashi, N. Fujii, N, Molecular imprinted nylon-6 as a recognition material of amino acids, Eur. Polym. J. 38 (2002) 521-529.

[29] N. Dencheva, J. Braz, T.G. Nunes, F.D. Oliveira, Z. Denchev, One-pot low temperature synthesis and characterization of hybrid poly(2-pyrrolidone) microparticles suitable for protein immobilization, Polymer 145 (2018) 402-4015.

[30] X. Li, B. Zhang, W. Li, X. Lei, X. Fan, L. Tian, H. Zhang, Q. Zhang, Preparation and characterization of bovine serum albumin surface-imprinted thermosensitive mag netic polymer microsphere and its application for protein recognition, Biosens. Bioelectronic 51 (2014) 261-267.

[31] O.B. Ptitsyn, in: C.B. Anfinsen, J.T. Edsall, F.M. Richards, D.S. Eisenberg (Eds.), Advances in Protein Chemistry, vol 47, Academic Press, New York, 1994, p. 171.

[32] N. Kim, J.H. Kim, S.W. Nam, B.S. Jeon, Y.J. Kim, Preparation of nylon 4 microspheres via heterogeneous polymerization of 2-pyrrolidone in a paraffin oil continuous Phase, J. Ind. Eng. Chem. 28 (2015) 236-240. 
[33] K. Hashimoto, Ring-opening polymerization of lactams. Living anionic polymerization and its applications, Prog. Polym. Sci. 25 (2000) 1411-1462.

[34] K. Tachibana, K. Hashimoto, N. Tansho, H. Okawa, Chemical modification of chain end in nylon 4 and improvement of its thermal stability, J. Polym. Sci. Part A: Polym. Chem. 49 (2011) 2495-2503.

[35] Z. Hua, Sh. Zhou, M. Zhao, Fabrication of a surface imprinted hydrogel shell over silica microspheres using bovine serum albumin as a model protein template,
Biosens. Bioelectron. 25 (2009) 615-622.

[36] C.H. Giles, D. Smith, A. Huitson, A general treatment and classification of the solute adsorption isotherm, I. Theoretical, J. Coll. Inter. Sci. 47 (1974) 755-765.

[37] T.S. Anirudhan, S. Sandeep, Synthesis and characterization of molecularly imprinted polymer of N-maleoylchitosan-grafted-2-acrylamido-2-methylpropanesulfonic acid and its controlled delivery and recognition of bovine serum albumin, Polym. Chem. 2 (2011) 2052-2061. 